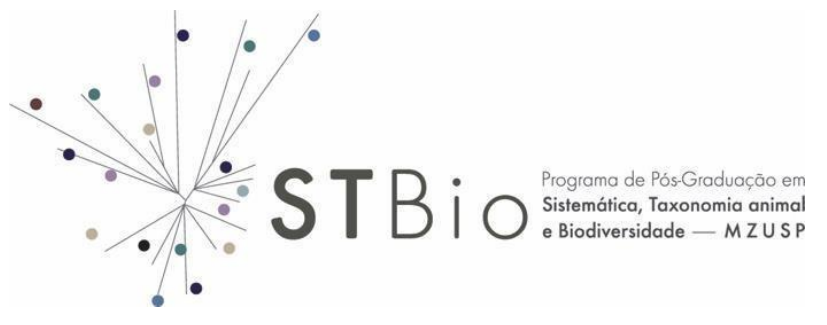

Tamires de Oliveira

Andrade

\begin{abstract}
AN INTEGRATIVE APPROACH TO DELIMIT SPECIES IN THE EULAEMA CINGULATA AND EULAEMA PSEUDOCINGULATA PAIR (HYMENOPTERA: APIDAE) USING MORPHOMETRIC AND MOLECULAR EVIDENCE

UMA ABORDAGEM INTEGRATIVA PARA DELIMITAR ESPÉCIES NO PAR EULAEMA CINGULATA E EULAEMA PSEUDOCINGULATA (HYMENOPTERA: APIDAE) UTILIZANDO EVIDÊNCIA MORFOMÉTRICA E MOLECULAR
\end{abstract}


Tamires de Oliveira

Andrade

\title{
An integrative approach to delimit species in the Eulaema cingulata and Eulaema pseudocingulata pair (Hymenoptera: Apidae) using morphometric and molecular evidence
}

\begin{abstract}
Uma abordagem integrativa para delimitar espécies no par Eulaema cingulata e Eulaema pseudocingulata (Hymenoptera: Apidae) utilizando evidência morfométrica e molecular
\end{abstract}

Original Version

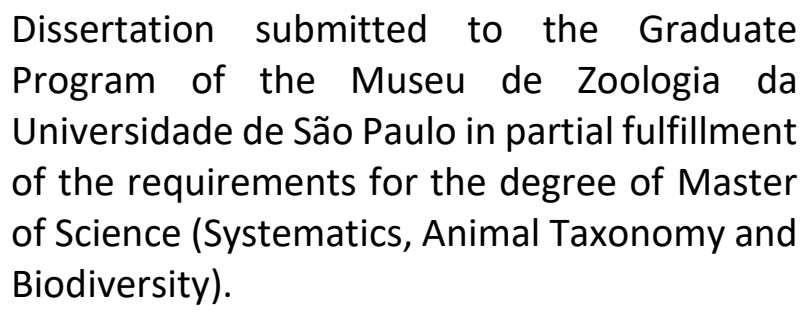

Advisor: Prof. Dr. Carlos Roberto Ferreira Brandão

Co-advisor: Dr. Kelli dos Santos Ramos 


\author{
Catalogação na Publicação \\ Serviço de Biblioteca e Documentação \\ Museu de Zoologia da Universidade de São Paulo
}

Andrade, Tamires de Oliveira

An integrative approach to delimit species in the Eulaema cingulata and Eulaema pseudocingulata pair (Hymenoptera: Apidae) using morphometric and molecular evidence.= Uma abordagem integrativa para delimitar espécies no par Eulaema cingulata e Eulaema pseudocingulata (Hymenoptera:Apidae) utilizando evidência morfométrica e molecular/ Tamires de Oliveira Andrade; orientador Carlos Roberto Ferreira Brandão; co-orientadora Kelly dos Santos Ramos. São Paulo, 2021.

$51 p$.

Dissertação de Mestrado - Programa de Pós-Graduação em Sistemática, Taxonomia e Biodiversidade, Museu de Zoologia, Universidade de São Paulo, 2021.

Versão original

Volume único

1. Hymenoptera - Apidae. 2. Eulaema cingulata.3. Eulaema pseudocingulata.. I. Brandão, Carlos Roberto Ferreira, orient.; II. Ramos, Kelly dos Santos, co-orinetadora. III.Título.

CDU 595.79 
Andrade, Tamires de $\mathrm{O}$.

An integrative approach to delimit species in the Eulaema cingulata and Eulaema pseudocingulata pair (Hymenoptera: Apidae) using morphometric and molecular evidence

Uma abordagem integrativa para delimitar espécies no par Eulaema cingulata e Eulaema pseudocingulata (Hymenoptera: Apidae) utilizando evidência morfométrica e molecular

Dissertation submitted to the Graduate Program of the Museu de Zoologia da Universidade de São Paulo in partial fulfillment of the requirements for the degree of Master of Science (Systematics, Animal Taxonomy and Biodiversity).

Date approved:

Deixar todos os espaços abaixo para preenchimento à mão.

COMMITTEE MEMBERS

Deletar as 4 últimas linhas no caso de bancas de Mestrado

Deletar esse box de instruções no documento final

Prof. Dr.

Institution:

Decision:

Signature:

Prof. Dr.

Institution:

Decision:

Signature:

Prof. Dr.

Institution:

Decision:

Signature: 
To my family for all support and trust. All thanks and this dedicatory will still be insufficient. 


\section{ACKNOWLEDGEMENTS}

Although this work was mainly developed by me, it is in fact the result of a collective work. It would not exist if it were not for the collaboration, even indirect, of countless people to whom I will be forever grateful.

I begin by thanking my parents, Adeilza and Joaquim, my favorite brother Elton and my dear uncle Jean, for always believing and trusting in me, for all support provided, for all love and dedication, and for always being available to help me with what it was necessary. Thank you for teaching me so much every day; I am a little piece of each one of you.

To my advisor, Professor Carlos Brandão for allowing me to study in the laboratory associated with one of the most important entomological collections in Brazil. For his valuable consideration in this and all my drafts and for arranging the financial support that made possible my travel to USA. Thank you for always being ready to help me and to be willing to contribute in the best possible way.

To my co-advisor, Dr. Kelli Ramos a thousand thanks. I thank her for all guidance over the years at the MZUSP. I thank her for trusting in my ability and for seeing in me someone to invest her time and knowledge in. I thank her for being always willing to help me, even if at eleven o'clock on a Sunday night. I thank her for her patience always (or almost always). I thank her also for the scolding me, without it I would not have learned that much, and also if I did not have her professional advice and for introducing me to her "abelhudos" friends. Thanks to her confidence I was able to let my mind fly away and conduct a study that I consider ambitious for a master's degree. I believed in my ability because she believed first in me. Thank you, dear Kelli, for not letting me give up. I will be forever grateful for you taking me to your home and for welcoming me with open arms by your family in one of the most difficult moments of the development of this work. Thank you for always trying to help me. Thank you for always being there and being an exceptional mentor. I learned a lot from you.

To Dr. Margarita López-Uribe for accepting to collaborate with this work and for receiving me in her laboratory. Thank you, Dr. Margarita for your valuable contributions, for your patience in teaching me how to use Phyluce and sometimes deciphering countless problems, when I got lost and had no idea on what to do next. Thank you for providing one of the most important stages of this work. The Penn State period was certainly one of the most memorable moments of my life.

I thank Michael Branstetter for the generation of the genomic data used in this work, for the "mini workshop" on analysis in Phyluce and for all the clarification of my doubts. 
Among the people who are indirectly linked to the development of this work, I want to emphasize one, which I titled my co-co-advisor: José Eduardo Serrano-Villavicencio, my beloved friend Pepe. I will be forever grateful to life for having given me the luck to meet someone as generous as you. Thank you for always being willing to read my texts and make your considerations. Thank you for having the patience to teach me morphometry from scratch, and sometimes explain the same thing to me more than once. Your contributions and incentives were fundamental to the development of this work. Thank you for all the conversations and advice. Thank you for being present, even at 7,681 Km of distance; even when I traveled abroad I did not feel completely alone because I knew that at any time and in any situation, I could count on you. I am sure you were one of the best gifts that graduate school has given to me.

To Sergio Bolivar, a core piece for the genomic analysis I performed at MZUSP. Thank you so much your willingness to always help me, opening time in your agenda to teach me how to use the cluster, how to run the programs and explain to me what that bunch of numbers meant. Your help was essential to the completion of this project. Thank you also for the conversations and coffee at the Ornito's laboratory.

My dear friends Mônica Ulysséa and Lívia Pires, for their support, confidence, and for being wonderful women and scientists who have always helped me, comforted me, calmed me, whether in the development of this work or in my life outside the walls of the MZUSP. Thank you for the countless conversations in our daily snack hour, whether they are about work or other matters. You girls certainly made the development of this work much lighter and smoother. Thanks Mônica for teaching me to be a field biologist and always remembering the essential items in an collecting outlet. I will never forget it. Thanks to Lívia for the moments of companionship, complaints, indignations, but also laughter and "imaginary newsstands". Thank you for including me in your projects and trusting that together we are stronger.

To my great friend Camila Pereira, for being so generous and staying awake with me at different times at her home, when she needed to arrive early or leave the laboratory late. Thank you for your always open arms to receive me and for being such an incredible person.

Thanks to all the members of the Lopez-Uribe's Lab for welcoming me so well at the Penn State University. I would like to thank particularly Dr. Nathaniel Pope, Shelby Kilpatrick, and Stephania Sandoval for their support in the use of genomic analysis programs. I would like to thank Stephania for having 'adopted me' during my time at State College. Thank you for all the conversations, for the help with Phyluce, and especially for being my translator at different times.

I thank Jaqueline Batillana for the technical support provided in the molecular biology 
laboratory of the MZUSP; Dione Seriperri and Marta Zamana for always being so efficient and helpful in the search for references and Marta Grobel for the quick solutions to bureaucratic questions I had during the development of the project.

To Luz Eneida, for the conversations and clarifications on phylogenomics and for helping me with part of the analysis of the UCEs.

To all researchers from other institutions that made donations or loans available of the material that was essential for the development of this study. Among them, Professor Elder Morato, from the Federal University of Acre (UFAC), Professor Fernando Silveira, from the Federal University of Minas Gerais (UFMG), Professor Márcio Oliveira, from the National Institute for the Amazon Research (INPA), Professor Orlando Tobias, from Museu Paraense Emílio Goeldi (MPEG), Professor Maria Cristina Gaglianone, from the State University of the North Fluminense Darcy Ribeiro (UENF). I am also grateful to Beatriz Coelho for having selected and sent to me the MPEG material, and to José Eustáquio, from the UFMG, who patiently helped me search for the copies in the collection.

To Thiago Polizei, for his friendship and companionship. Thank you for the trips, congresses, lunches, and for all the ever present laughter. To my dear friends of Laboratory: Alvaro and Helena, for all the advice, outburst, and coffee with small talk. To MZUSP's friends: Talita Roell and Juan Pablo, for always being willing to help me with whatever was necessary. To Rafaela Vendramel for her companionship at different times at MZUSP, for her conversations and for always being sincere and fair. Thank you to all these dear friends for all the moments we spent together and for making my days at the MZUSP more joyful.

I am grateful to all the other colleagues that I made at the MZUSP, and that were also directly important in the development of this work. Without the conversations and laughter, in the disciplines, at lunches or at $4 \mathrm{pm}$ coffee, the development of this dissertation would have been much more painful.

I am grateful to the Conselho Nacional de Desenvolvimento Científico e Tecnológico $(\mathrm{CNPq})$, which, through funding from the Programa de Apoio a Projetos de Pesquisas para a Capacitação e Formação de Recursos Humanos em Taxonomia Biológica - PROTAX, made possible my travel to the United States for the realization of the genomic analyzes.

This study was financed in part by the Coordenação de Aperfeiçoamento de Pessoal de Nível Superior - Brasil (CAPES) - Finance Code 001. 
"Penso no que faço, com fé. Faço o que tenho que fazer, com amor. Eu me esforço para ser melhor, pois bondade também se aprende. Mesmo quando tudo parecer desabar cabe a mim decidir entre rir e chorar, ir ou ficar, desistir ou lutar; porque descobri, no caminho incerto da vida que o mais importante é decidir." Cora Coralina 


\begin{abstract}
The study of species delimitation has been a rich scientific field that makes use of different sources of data in order to identify independently evolving lineages that might be recognized as species. Males of the solitary orchid bees Eulaema cingulata (Fabricius, 1804) and Eulaema pseudocingulata Oliveira, 2006 are morphologically similar, although differ in the shape of the velvety area of the middle leg. These nominal species are relatively commonly recorded in bee diversity studies in Brazil; they have been, however, recognized by conflicting hypotheses of interspecific delimitation. Here we investigate the limits of both nominal species combining distinct methods: geometric morphometrics (GM), phylogenetics, COI-barcoding and pairwise genetic p-distance with a species delimitation method based on multispecies coalescent. We obtained data from 126 representative specimens of the entire geographical range in which the nominal species occur and assess species boundaries under the general lineage concept. We found substantial overlapping in the shape of forewings and heads of species in the GM analysis. The results of COI-barcoding analysis showed pair-wise genetic distances lower than $3 \%$ (within E. cingulata $1.3 \%$ and within E. pseudocingulata $0.7 \%$, while between the two species $0.95 \%$ ). The Bayesian phylogenetic reconstruction clustered both species in a strongly supported monophyletic group, however, these forms were not reconstructed as distinct clades. The sequencing of UCEs recovered 2.180 homologous loci that provided no sufficient variability to recognize both forms either as different species or as independent evolutionary units. Our results support the recognition of E. cingulata and E. pseudocingulata as members of the same evolutionary unit.
\end{abstract}

Keywords: Barcoding. Euglossini. Taxonomy. Orchid Bees. Phylogenomics. Species Delimitation. Ultraconserved Elements. 


\section{RESUMO}

O estudo de delimitação de espécies tem sido um fértil campo da ciência, que faz uso de diferentes fontes de dados para identificar linhagens evolutivas independentes que devam ser reconhecidas como espécies. Os machos das espécies de abelhas solitárias das orquídeas Eulaema cingulata (Fabricius, 1804) e Eulaema pseudocingulata Oliveira, 2006 têm sido reconhecidas como entidades diferentes na literatura, são morfologicamente similares e se diferenciariam pela forma da região aveludada da perna média. Registros dessas espécies nominais são relativamente comuns em estudos de diversidade de abelhas no Brasil; no entanto, estes nomes têm sido reconhecidos a partir de hipóteses conflitantes de delimitação interespecífica. Aqui nós investigamos se existem limites entre ambas as espécies combinando métodos como Morfometria Geométrica (MG), filogenética, COI-barcoding e distância genética com um método de delimitação de espécies baseado em coalescência de múltiplas espécies. Obtivemos dados de 126 espécimes representativos de toda a distribuição geográfica em que as espécies nominais ocorrem e avaliamos os limites das espécies sob o conceito de linhagem geral. Encontramos sobreposições substanciais na forma das asas anteriores e das cabeças na análise de MG. Os resultados da análise do COI-barcoding mostraram distâncias genéticas inferiores a 3\% (dentro de E. cingulata 1,3\% e dentro de E. pseudocingulata 0,7\%, enquanto entre as duas espécies $0,95 \%$ ). A reconstrução filogenética bayesiana agrupou ambas as formas em um grupo monofilético fortemente suportado, apesar delas não terem sido reconstruídas, entretanto, como clados distintos. O sequenciamento de UCEs recuperou 2.180 loci homólogos que corroboram com análises anteriores e não forneceram variabilidade suficiente para reconhecer ambas as formas como espécies biológicas diferentes ou como unidades evolutivas independentes. Nossos resultados apoiam o reconhecimento de ambas as formas como membros de uma mesma unidade evolutiva.

Palavras-chave: Abelhas das Orquídeas. Barcoding. Delimitação de Espécies. Elementos Ultraconservados. Euglossini. Filogenômica. Taxonomia. 


\section{CONTENTS}

$\begin{array}{ll}\text { 1. INTRODUCTION } & 13\end{array}$

2. MATERIAL AND METHODS 18

$\begin{array}{lr}\text { 2.1. Sampling and DNA extraction } & 18\end{array}$

2.2. Morphometric data $\quad 25$

2.3. Amplification and sequencing of COI $2 \mathbf{2 6}$

2.4. UCE library preparation $\quad 26$

$\begin{array}{lr}\text { 2.5. Bioinformatics } & 28\end{array}$

2.6. Molecular approaches for species phylogeny and delimitation $\quad \mathbf{2 8}$

3. RESULTS 29

3.1. Morphometric geometrics 29

3.2. Genetic distance $\quad 31$

3.3. Phylogenetic relationships $\quad 31$

4. DISCUSSION 34

$\begin{array}{ll}\text { 5. CONCLUSIONS } & 37\end{array}$

$\begin{array}{lr}\text { 6. REFERENCES } & 38\end{array}$ 


\section{INTRODUCTION}

Taxonomic identification is a critical step in biodiversity studies and should be the starting point for further detailed research, since species' misidentifications or the application of ambiguous species concepts can carry serious negative impacts, hindering the investigation of any biological phenomenon (Austen et al., 2016). Species that diverged recently may exhibit a very similar morphology and can be misclassified under an erroneous species name, leading to an underestimation of biological diversity (Leliaert et al., 2014). On the other hand, conspecific individuals can display discrete variation in morphological characters (e. g. Quezada et al., 2015; Lepeco \& Gonçalves, 2018). When these variable characters are used for species identification, the same evolutionary unit may be assigned to different species, overestimating the biodiversity (Sivogini et al., 2016).

To circumvent some of the limitations of taxonomy by using exclusively morphology (Padial et al., 2010; Jorger \& Schorodi, 2013), integrative taxonomy incorporates other approaches, such as geometric morphometrics, to assess intra- and inter-specific variation to facilitate the recognition of different species (Francisco et al., 2008; Francoy et al., 2012; Quezada-Euán et al., 2015), and molecular biology techniques to complement species identification and delimitation based before solely on morphology (Blaxter, 2004; Goldstein \& DeSalle, 2010). Molecular markers are an alternative tool for the characterization of different species (Blaxter, 2004). The identification of animal biological diversity using molecular markers has been mainly demonstrated through the use of the mitochondrial gene cytochrome c oxidase subunit 1 (COI). This gene has been used as a genetic barcode to identify taxa because it can be easily amplified by using universal primers (Herbert et al., 2003), displays relatively high substitution rate suitable for constructing a phylogenetic tree (Galtier et al., 2009), suffers comparatively rapid evolution of differentiation between closely related species, and is likely involved in the process of speciation (Hill, 2016). DNA barcoding is a common molecular method in the identification and delimitation of species from various groups, from invertebrates (Freitas et al., 2018; Grando et al., 2018) to birds (Herbert et al., 2004). This method gained increased acceptance due to being simple and affordable, and to promise a practical, standardized, species-level identification tool that can be used for biodiversity assessment (Kress et al., 2005; Padial \& De La Riva, 2007). In a species identification analysis using a barcoding approach, within-species DNA sequences need to be more similar to one another than to sequences in different species (Ward et al., 2005). Genetic distances between COI 
sequences from individuals are calculated and values higher than $3 \%$ divergence has been commonly used as indicative of the presence of different species (Herbert et al., 2003).

The application of DNA barcoding approach to systematics has revolutionized the discovery and description of biodiversity and its incorporation to taxonomic classification can help to solve some of the problems of relying only on morphological classification (Grando et al., 2018). Basing a species description on a variety of characters from different and independent datasets is generally regarded as the best practice (Dayrat, 2005; DeSalle et al., 2005). Integrative taxonomy is capable to recognize species which are identifiable by just a few apparent morphological criteria once their existence is revealed by other methods (Lajus et al., 2015; Sáez \& Lozano, 2005). When species are considered as independently evolving lineages (de Queiroz, 2007), different lines of evidence are additive to each other. Molecular evidence can provide an independent test of morphological assessments of species identity and vice versa (Page et al., 2005). These combined approaches are a powerful tool to detect cryptic species, to clarify species boundaries, to estimate species diversity, including of bees (e.g. Gibbs, 2009; González-Vaquero et al., 2019; Pauly et al., 2019), and can also contribute to the estimation of true richness and hence to synonymize illegitimate nominal species (e. g. González-Vaquero \& Roig-Alsina, 2019).

Evolutionary inference from single-locus data presents some limitations, however, including the retention of ancestral polymorphism and incomplete lineage sorting. This type of data represents the history of a single gene that might not be representative of a species history, which can lead to ambiguities in assessing species boundaries (Hickerson et al., 2006; Knowles \& Carstens, 2007; Leliaert et al., 2014). More recently, multi-locus genetic data has provided potential evidence of divergence even at an early stage of diversification (Knowles \& Carstens, 2007). Methods for multi-marker species delimitation have been developed mainly as a response to technical advances in sequencing technology that allow for the generation of large-scales datasets (Faircloth et al., 2012; Lemmon et al., 2012; Lemmon \& Lemmon, 2013). The advent of Massively Parallel Sequencing (MPS) and the development of generalized data collection facilitated the acquisition of multi-locus datasets for large numbers of individuals and have been an important tool to resolve the interrelationships of longstanding problematic taxa (Crawford et al., 2012; Faircloth et al., 2012; McCormack et al., 2013).

Ultraconserved Elements (UCEs) markers have been recently developed and can be used in conjunction with sequence capture and MPS to generate large amounts of orthologous sequence data among a taxonomically diverse set of species (Faircloth et al., 2012). UCEs were originally discovered in mammals (Bejerano et al., 2004) but are common in a diversity of 
Arthropods (Blaimer et al., 2016; Faircloth et al., 2015; Longino \& Branstetter, 2020). These elements are short stretches of highly conserved DNA shared across distantly related taxa, which harbor variation in its flanking regions. The variation in the flanks increases with distance from the core UCE, providing information for estimating phylogenies at multiple evolutionary timescales (Smith et al., 2014; Crawford et al., 2015; Faircloth et al., 2012). Because of the increase in variation in the flanking regions of UCEs, these markers are suitable for studies at narrow timescales. One of the advantages of using UCEs is that they work with suboptimally preserved specimens and/or degraded DNA (McCormack et al., 2015; Blaimer et al., 2016).

With multilocus genetic data, coalescent models have been implemented to identify the evolutionary processes that contribute to speciation (Fujita et al., 2012). Coalescence-based methods highlight incomplete lineage-sorting as a source of inconsistency between gene trees and the species trees (Rannala \& Yang, 2003; Liu et al., 2009; Heled \& Drummond, 2010), inferring species limits based on a rigorous population framework (Fujita et al., 2012; Rannala, 2015). With the addition of data sources, coalescent-based species delimitation might play an important role in an integrative taxonomy that emphasizes the identification of species and the processes that have promoted lineage diversification (Fujita et al., 2012). The development of species delimitation methods that are based on the multispecies coalescent model (MSC) was an important advance in biodiversity research (Takahata et al., 1995; Rannala \& Yang, 2003). Several studies have delimited species using this approach (e. g. Carstens \& Dewey, 2010; Leaché \& Fujita, 2010; Carstens \& Satler, 2013).

Morphometric and molecular approaches have been successfully applied in studies of bees' biogeography, systematic and evolution (Quezada-Euán et al., 2007; Quezada-Euán et al., 2015; Santos et al., 2019). However, there are relatively few studies using integrative taxonomy to assess the relationships and species thresholds in euglossine bees. Orchid bees (Apidae: Euglossini) are endemic bees of the Neotropical Region, so-called because of the strict relationship of males with most orchids in this region, and are also known because of their brilliant metallic integumental coloration (Michener, 2007). Males of orchid bees collect aromatic substances found in several plant species, especially orchids, and nonfloral resources (Dressler, 1982) to use these substances principally as a signal to attract females and communicate availability for mating (Eltz et al., 2011). Five Euglossini genera are recognized: Euglossa Latreille 1802, Eufrisea Cockerell 1908, Eulaema Lepeletier 1841, Exaerete Hoffmannsegg 1817, and Aglae Lepeletier \& Serville 1825, encompassing approximately 250 species (Michener, 2007; Moure et al., 2012). Eulaema contains the largest species of orchid bees, with body size varying from 18 to 30mm in length (Oliveira, 2000; Melo, 2014), and do 
not exhibit a metallic shine in the head and thorax as many other Euglossini do (Oliveira, 2000).

The species Eulaema (Apeulaema) cingulata (Fabricius, 1804) has a wide distribution associated mainly to forested areas in the Neotropical region, being frequently sampled in faunistic inventories and widely used for ecological studies (e.g. Cavalcante et al., 2012, RochaFilho \& Garófalo, 2015, Marques et al., 2017). However, this name is involved in conflicting hypotheses of interspecific delimitation with Eulaema (Apeulaema) pseudocingulata Oliveira, 2006. This second nominal species was described based on few morphological differences from E. cingulata and due to its geographic distribution, apparently restricted to the Amazon Forest (Oliveira, 2006). The taxonomic status of Eulaema cingulata and E. pseudocingulata has been subject of debate. Males of both species can only be separated phenotypically by the velvety area on the middle leg, which is much narrower and far from the posterior edge in Eulaema pseudocingulata, whereas in E. cingulata it is wide and close to the posterior edge. In addition, the coloration of the male abdomen is relatively darker in E. pseudocingulata (Figure 1), which description was carried out based only on morphological aspects of males as the female is unknown. In the present study we evaluate the differences between specimens identified as Eulaema cingulata and Eulaema pseudocingulata, employing an integrative taxonomic approach that combines morphological and molecular data in order to test species identities. Our expectation was to clarify objectively whether different morphs correspond to one or two evolutionary units. 


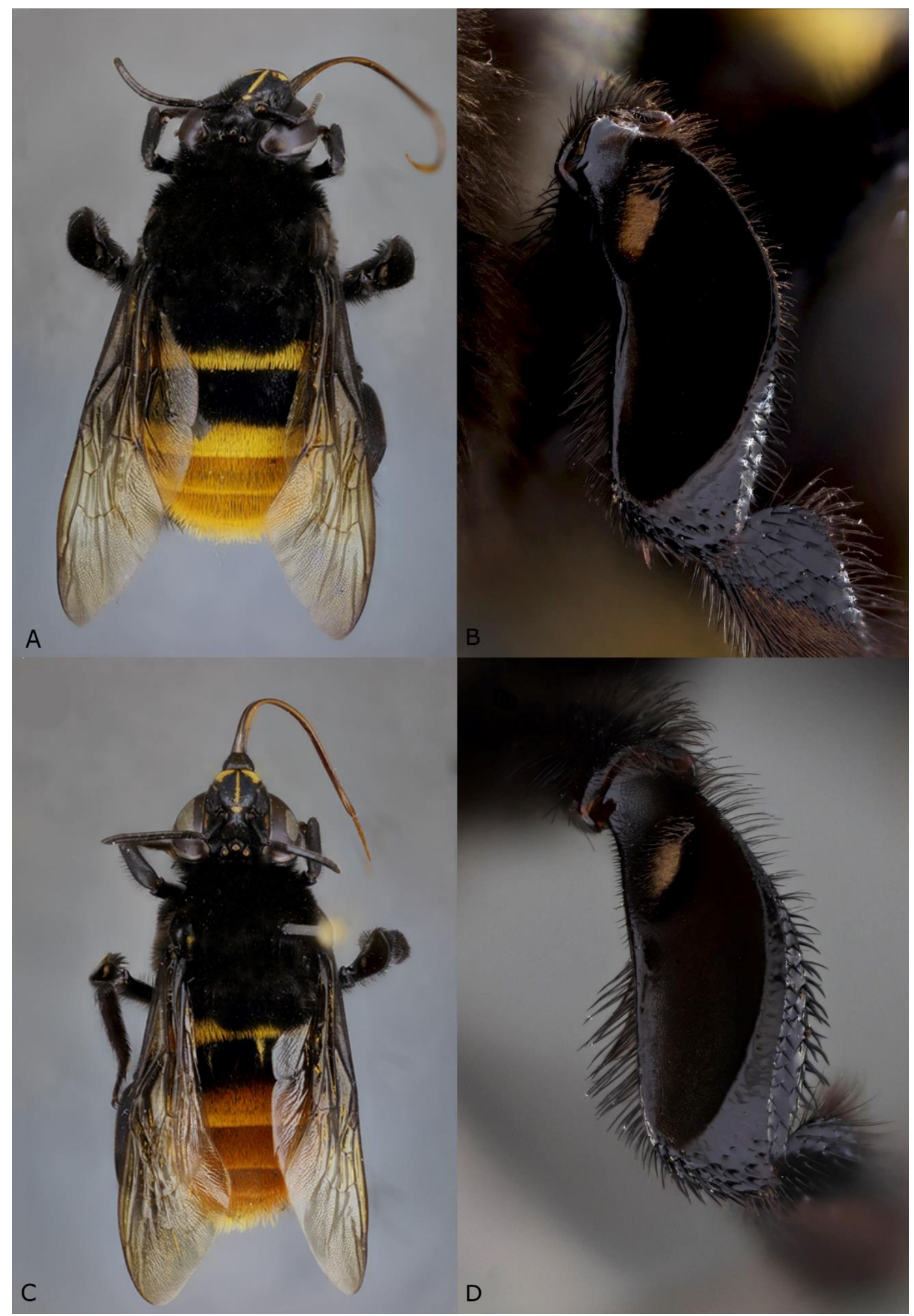

Figure 1. A-B. Male specimens of Eulaema cingulata from Sergipe, Brazil. (A) Body in dorsal view. (B) Velvety area in the mid tibiae. C-D. Male specimens of E. pseudocingulata from Pará, Brazil. (C) Body in dorsal view. (D) Velvety area in the mid tibiae. 


\section{MATERIAL AND METHODS}

\subsection{Sampling and DNA extraction}

The material selected for the present study covers most of the reported distribution of Eulaema cingulata and E. pseudocingulata, especially in the areas they appear to be sympatric (Amazon Forest). A total of 107 male specimens were used for the Geometric Morphometrics analysis, being 53 and 54 specimens of E. pseudocingulata and E. cingulata, respectively. For molecular analyses we obtained DNA sequences from 19 recently-collected males, maximum age of 10 years after collection, used in COI $(n=8)$ and UCE $(n=15)$ analyses, while some samples were used in both molecular approaches $(n=4)$. Four sequences of the mitochondrial gene Cytochrome oxidase I (COI) were obtained from GenBank and two sequences were obtained from BOLD Systems. We added five other Eulaema species as outgroups in our molecular analyses: E. mocsaryi and E. nigrita for COI analysis, and E. bombiformis, E. chocoana, E. meriana, E. mocsaryi and E. nigrita for the phylogenomic analysis. Information of the specimen included, and their repositories, are presented in Table 1.

We extracted total genomic DNA from the hind legs or thoracic muscles of the specimens using Qiagen DNeasy Kits (Qiagen ${ }^{\mathrm{TM}}$ ), including modifications from Evangelista (2012). Some specimens used were directly preserved in $100 \%$ ethanol right after collecting, whereas others were kept dried mounted for various periods of time. Before extraction, pinned specimens were left in a humid chamber for 24 hours in order to facilitate the removal of the leg or abdomen muscle without damaging the whole specimen. Then the samples were digested with buffer plus $20 \mu \mathrm{L}$ of protease $\mathrm{K}$ and after six hours we added more $10 \mu \mathrm{L}$. The incubation was performed overnight (up to 12 hours). 
Table 1. Voucher ID, locality data and institutional repositories of the specimens used in the present study. INPA = Instituto Nacional de Pesquisas da Amazônia; MZUSP = Museu de Zoologia da Universidade de São Paulo; UENF = Universidade Estadual do Norte Fluminense Darcy Ribeiro; UFAC = Universidade Federal do Acre; UFMG = Universidade Federal de Minas Gerais.

\begin{tabular}{|c|c|c|c|c|c|c|c|c|c|c|}
\hline Species & Country & $\begin{array}{c}\text { State/ } \\
\text { Province }\end{array}$ & Latitude & Longitude & Date & \multicolumn{2}{|c|}{$\begin{array}{c}\text { Geometric } \\
\text { morphometrics }\end{array}$} & COI & UCEs & Repository \\
\hline E. bombiformis & Ecuador & Orellana & $0^{\circ} 27^{\prime} 16.26^{\prime \prime S}$ & $76^{\circ} 59^{\prime} 42.10^{\prime \prime} \mathrm{W}$ & 02.viii.2009 & - & - & - & ML256 & $\begin{array}{c}\text { López-Uribe } \\
\text { lab. }\end{array}$ \\
\hline E. chocoana & Colômbia & Chocó & $5^{\circ} 29^{\prime} 58.86 " \mathrm{~N}$ & $76^{\circ} 32^{\prime} 33.35^{\prime \prime} \mathrm{W}$ & 18.vi.2003 & - & - & - & ML023 & $\begin{array}{c}\text { López-Uribe } \\
\text { lab. }\end{array}$ \\
\hline E. cingulata & Brazil & Acre & $09^{\circ} 59^{\prime} 14.3^{\prime \prime S}$ & $67^{\circ} 50 ' 29.0^{\prime \prime} \mathrm{W}$ & 25.i.2016 & EC011 & - & - & - & UFAC \\
\hline E. cingulata & Brazil & Acre & $07^{\circ} 26^{\prime} 45.20^{\prime \prime} \mathrm{S}$ & $72^{\circ} 54^{\prime} 37.20^{\prime \prime} \mathrm{W}$ & 24.vi.2017 & EC010 & - & - & - & UFAC \\
\hline E. cingulata & Brazil & Alagoas & $09^{\circ} 12^{\prime} 42.1^{\prime \prime S}$ & $35^{\circ} 52^{\prime} 12.0^{\prime \prime} \mathrm{W}$ & 13.v.2015 & EC016 & EC016 & - & - & UFMG \\
\hline E. cingulata & Brazil & Alagoas & $09^{\circ} 12^{\prime} 42.1^{\prime \prime S}$ & $35^{\circ} 52^{\prime} 12.0^{\prime \prime} \mathrm{W}$ & 13.v.2015 & EC015 & EC015 & - & - & UFMG \\
\hline E. cingulata & Brazil & Amazonas & - & - & - & EC043 & - & - & - & INPA \\
\hline E. cingulata & Brazil & Amazonas & - & - & ix.1992 & EC039 & - & - & - & INPA \\
\hline E. cingulata & Brazil & Amazonas & $03^{\circ} 56^{\prime} 02^{\prime \prime S}$ & $61^{\circ} 19^{\prime} 03^{\prime \prime} \mathrm{W}$ & $\begin{array}{c}\text { 29.xi- } \\
\text { 08.xii.2003 }\end{array}$ & EC042 & - & - & - & INPA \\
\hline E. cingulata & Brazil & Amazonas & $3^{\circ} 07^{\prime} 11.69^{\prime \prime} \mathrm{S}$ & $60^{\circ} 01^{\prime} 14.22^{\prime \prime} \mathrm{W}$ & 22.vii.2012 & EC045 & - & - & - & INPA \\
\hline E. cingulata & Brazil & Amazonas & 6³0'24.84"S & $64^{\circ} 33^{\prime} 05.4^{\prime \prime} \mathrm{W}$ & 07.v.2013 & EC044 & - & - & - & INPA \\
\hline E. cingulata & Brazil & Amazonas & $4^{\circ} 05^{\prime} 35.47^{\prime \prime} \mathrm{S}$ & $63^{\circ} 08^{\prime} 25.05^{\prime \prime} \mathrm{W}$ & 16-17.vi.2017 & EC046 & - & - & - & INPA \\
\hline E. cingulata & Brazil & Bahia & $15^{\circ} 47^{\prime} 45.7^{\prime \prime} \mathrm{S}$ & $40^{\circ} 31^{\prime} 57.0^{\prime \prime} \mathrm{W}$ & 30.i.2014 & EC033 & EC033 & - & - & UFMG \\
\hline E. cingulata & Brazil & Bahia & $17^{\circ} 06^{\prime} 18.6^{\prime \prime S}$ & $39^{\circ} 20^{\prime} 18.1 " \mathrm{~W}$ & 12.xii.2015 & EC032 & EC032 & - & - & UFMG \\
\hline E. cingulata & Brazil & Bahia & $16^{\circ} 52^{\prime} 48.9^{\prime \prime} \mathrm{S}$ & $39^{\circ} 24^{\prime} 48.2^{\prime \prime} \mathrm{W}$ & 14.xii.2015 & EC031 & EC031 & - & - & UFMG \\
\hline E. cingulata & Brazil & $\begin{array}{l}\text { Espírito } \\
\text { Santo }\end{array}$ & $\begin{array}{l}19^{\circ} 06^{\prime-} \\
19^{\circ} 18^{\prime \prime S}\end{array}$ & $39^{\circ} 45^{\prime}-40^{\circ} 19^{\prime} \mathrm{W}$ & 24.iv.1997 & EC049 & - & - & - & INPA \\
\hline
\end{tabular}


Table 1. Voucher ID, locality data and institutional repositories of the specimens used in the present study (continued).

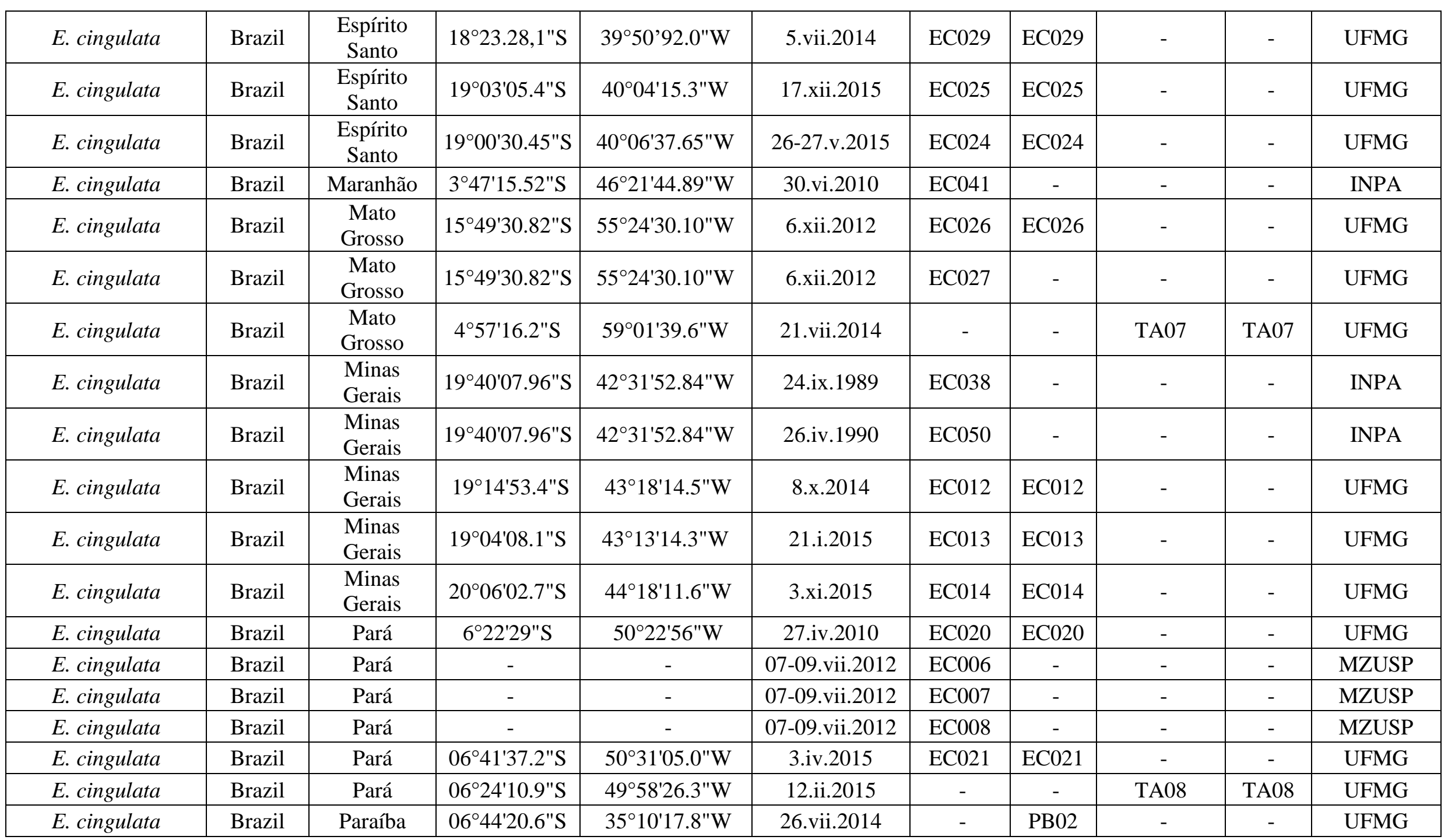


Table 1. Voucher ID, locality data and institutional repositories of the specimens used in the present study (continued).

\begin{tabular}{|c|c|c|c|c|c|c|c|c|c|c|}
\hline E. cingulata & Brazil & Paraíba & $06^{\circ} 42^{\prime} 48.5^{\prime \prime} \mathrm{S}$ & $35^{\circ} 11^{\prime} 46.8^{\prime \prime} \mathrm{W}$ & 27.vii.2014 & EC028 & EC028 & - & - & UFMG \\
\hline E. cingulata & Brazil & Paraíba & $06^{\circ} 43^{\prime} 18.9^{\prime \prime} \mathrm{S}$ & $35^{\circ} 12^{\prime} 13.3^{\prime \prime} \mathrm{W}$ & 27.vii.2014 & - & - & - & TA09 & UFMG \\
\hline E. cingulata & Brazil & $\begin{array}{l}\text { Rio de } \\
\text { Janeiro }\end{array}$ & $21^{\circ} 47^{\prime} 23^{\prime \prime} \mathrm{S}$ & $41^{\circ} 26^{\prime} 15^{\prime \prime} \mathrm{W}$ & 26.ix.2008 & - & RJ02 & - & - & UENF \\
\hline E. cingulata & Brazil & $\begin{array}{l}\text { Rio de } \\
\text { Janeiro }\end{array}$ & $21^{\circ} 47^{\prime} 23^{\prime \prime S}$ & $41^{\circ} 26^{\prime} 15^{\prime \prime} \mathrm{W}$ & 26.ix.2008 & - & RJ02 & - & - & UENF \\
\hline E. cingulata & Brazil & $\begin{array}{l}\text { Rio de } \\
\text { Janeiro }\end{array}$ & $22^{\circ} 37^{\prime} 16.9^{\prime \prime S}$ & $43^{\circ} 47^{\prime} 05.0^{\prime \prime} \mathrm{W}$ & 24.ii.2012 & - & RJ01 & - & - & UFMG \\
\hline E. cingulata & Brazil & Rondônia & $9^{\circ} 35^{\prime} 24.00^{\prime \prime S}$ & $65^{\circ} 03^{\prime} 00.00^{\prime \prime} \mathrm{W}$ & 5.iii.2010 & $\mathrm{EC} 002$ & - & - & - & MZUSP \\
\hline E. cingulata & Brazil & Rondônia & - & - & 8.iii.2010 & - & EC001 & - & TA12 & MZUSP \\
\hline E. cingulata & Brazil & Rondônia & $9^{\circ} 36^{\prime} 00.00^{\prime \prime S}$ & $65^{\circ} 21^{\prime} 36.00^{\prime \prime} \mathrm{W}$ & 10.iii.2010 & EC003 & - & - & - & MZUSP \\
\hline E. cingulata & Brazil & Rondônia & $12^{\circ} 13^{\prime} 56.6^{\prime \prime S}$ & $61^{\circ} 24^{\prime} 24.9^{\prime \prime} \mathrm{W}$ & 6.xii.2012 & EC019 & EC019 & - & - & UFMG \\
\hline E. cingulata & Brazil & Rondônia & $12^{\circ} 13^{\prime} 56.6^{\prime \prime S}$ & $61^{\circ} 24^{\prime} 24.9^{\prime \prime} \mathrm{W}$ & 7.xii.2012 & $\mathrm{EC} 018$ & EC018 & - & - & UFMG \\
\hline E. cingulata & Brazil & Sergipe & $10^{\circ} 45^{\prime} 26.7^{\prime \prime S}$ & $37^{\circ} 20^{\prime} 53.5^{\prime \prime} \mathrm{W}$ & 22.vii.2014 & $\mathrm{EC} 034$ & EC034 & - & - & UFMG \\
\hline E. cingulata & Brazil & Tocantins & $10^{\circ} 08^{\prime} \mathrm{S}$ & $48^{\circ} 20^{\prime} \mathrm{W}$ & 3.i.2015 & $\mathrm{EC} 036$ & - & - & - & INPA \\
\hline E. cingulata & Colômbia & Medellin & $6^{\circ} 13 ' 31.44^{\prime \prime} \mathrm{N}$ & $75^{\circ} 32^{\prime} 13.46^{\prime \prime} \mathrm{W}$ & i. 2014 & - & - & TA10 & TA10 & UFMG \\
\hline E. cingulata & Colômbia & Monteria & $8^{\circ} 42^{\prime} 54.96 " \mathrm{~N}$ & $75^{\circ} 51^{\prime} 48.21^{\prime \prime W}$ & i. 2014 & $\mathrm{CO} 01$ & $\mathrm{CO} 01$ & - & - & UFMG \\
\hline E. cingulata & $\begin{array}{l}\text { Costa } \\
\text { Rica }\end{array}$ & Cartago & $9^{\circ} 54^{\prime} 24.14 " \mathrm{~N}$ & $83^{\circ} 4048.18^{\prime \prime W}$ & 11.vi.1976 & EC048 & - & - & - & INPA \\
\hline E. meriana & $\begin{array}{l}\text { Costa } \\
\text { Rica }\end{array}$ & La Selva & $9^{\circ} 33^{\prime} 25.93 " \mathrm{~N}$ & $82^{\circ} 55^{\prime} 55.93^{\prime \prime W}$ & 06.vi.2008 & - & - & - & ML089 & $\begin{array}{c}\text { López-Uribe } \\
\text { lab. }\end{array}$ \\
\hline E. meriana & Peru & $\begin{array}{l}\text { Madre de } \\
\text { Dios }\end{array}$ & $12^{\circ} 28^{\prime} 50.11^{\prime S} \mathrm{~S}$ & $70^{\circ} 20^{\prime} 32.21^{\prime \prime W}$ & 3.xi.1997 & - & - & MN345153.1 & - & GenBank \\
\hline
\end{tabular}


Table 1. Voucher ID, locality data and institutional repositories of the specimens used in the present study (continued).

\begin{tabular}{|c|c|c|c|c|c|c|c|c|c|c|}
\hline E. mocsaryi & Colômbia & $\begin{array}{c}\text { Puerto } \\
\text { Carreño } \\
\end{array}$ & $6^{\circ} 11^{\prime} 23.68^{\prime \prime} \mathrm{N}$ & $67^{\circ} 28^{\prime} 57.25^{\prime \prime} \mathrm{W}$ & 2.x.2002 & - & - & EU421502 & - & GenBank \\
\hline E. pseudocingulata & Brazil & Acre & $9^{\circ} 58^{\prime} 31.21 " \mathrm{~S}$ & $68^{\circ} 25^{\prime} 45.56^{\prime \prime} \mathrm{W}$ & 03.ii.1997 & EC022 & EC022 & - & - & UFMG \\
\hline E. pseudocingulata & Brazil & Acre & $9^{\circ} 58 ' 31.21 " \mathrm{~S}$ & $68^{\circ} 25^{\prime} 45.56^{\prime \prime} \mathrm{W}$ & 03.ii.1997 & EP029 & EP029 & - & - & UFMG \\
\hline E. pseudocingulata & Brazil & Acre & $9^{\circ} 58^{\prime} 31.21 " \mathrm{~S}$ & $68^{\circ} 25^{\prime} 45.56^{\prime \prime} \mathrm{W}$ & 05.x.1997 & EP028 & EP028 & - & - & UFMG \\
\hline E. pseudocingulata & Brazil & Acre & $10^{\circ} 02^{\prime} 01.5^{\prime \prime} \mathrm{S}$ & $67^{\circ} 46^{\prime} 14.2^{\prime \prime} \mathrm{W}$ & 15.ix.2015 & EP021 & - & - & - & UFAC \\
\hline E. pseudocingulata & Brazil & Acre & $10^{\circ} 03^{\prime} 15.6^{\prime \prime} \mathrm{S}$ & $67^{\circ} 49^{\prime} 30.8^{\prime \prime} \mathrm{W}$ & 14.xii.2015 & EP015 & EP015 & - & - & UFAC \\
\hline E. pseudocingulata & Brazil & Acre & $10^{\circ} 02^{\prime} 01.5^{\prime \prime S}$ & $67^{\circ} 46^{\prime} 14.2^{\prime \prime} \mathrm{W}$ & 17.i.2016 & EP016 & EP016 & - & - & UFAC \\
\hline E. pseudocingulata & Brazil & Acre & $10^{\circ} 02^{\prime} 01.5^{\prime \prime S}$ & $67^{\circ} 46^{\prime} 14.2^{\prime \prime} \mathrm{W}$ & 17.i.2016 & - & - & TA01 & - & UFAC \\
\hline E. pseudocingulata & Brazil & Acre & $09^{\circ} 59^{\prime} 14.3^{\prime \prime S}$ & $67^{\circ} 50^{\prime} 29.0^{\prime \prime} \mathrm{W}$ & 25.i.2016 & EP007 & - & - & - & UFAC \\
\hline E. pseudocingulata & Brazil & Acre & $09^{\circ} 57^{\prime} 14.0^{\prime \prime} \mathrm{S}$ & $67^{\circ} 47^{\prime} 34.7^{\prime \prime} \mathrm{W}$ & 26.i.2016 & EP033 & EP033 & - & - & UFAC \\
\hline E. pseudocingulata & Brazil & Amapá & $0^{\circ} 01^{\prime} 44.25 " \mathrm{~N}$ & $51^{\circ} 04^{\prime} 03.96 " \mathrm{~W}$ & iii.1991 & EP050 & - & - & - & INPA \\
\hline E. pseudocingulata & Brazil & Amapá & $0^{\circ} 2 ' 56.35 " \mathrm{~S}$ & $51^{\circ} 7^{\prime} 45.45^{\prime \prime} \mathrm{W}$ & 31.iii.2012 & EP043 & - & - & - & INPA \\
\hline E. pseudocingulata & Brazil & Amazonas & $05^{\circ} 15^{\prime} 39^{\prime \prime} \mathrm{S}$ & $60^{\circ} 42^{\prime} 32^{\prime \prime} \mathrm{W}$ & 23.iv.2005 & EP041 & - & - & - & INPA \\
\hline E. pseudocingulata & Brazil & Amazonas & $07^{\circ} 19^{\prime} 10^{\prime \prime} \mathrm{S}$ & $64^{\circ} 40^{\prime} 07^{\prime \prime} \mathrm{W}$ & $\begin{array}{c}\text { 13.vi- } \\
\text { 07.vii.2006 } \\
\end{array}$ & EP044 & - & - & - & INPA \\
\hline E. pseudocingulata & Brazil & Amazonas & $00^{\circ} 25^{\prime} 09.3^{\prime \prime} \mathrm{N}$ & $63^{\circ} 23^{\prime \prime} 00.7 " \mathrm{~W}$ & 30.vii.2007 & EP040 & - & - & - & INPA \\
\hline E. pseudocingulata & Brazil & Amazonas & $2^{\circ} 57^{\prime} 48.04 " \mathrm{~S}$ & $59^{\circ} 55^{\prime} 22.20^{\prime \prime} \mathrm{W}$ & 14.viii.2008 & EP042 & - & - & - & INPA \\
\hline E. pseudocingulata & Brazil & Goiás & $18^{\circ} 15^{\prime} 38^{\prime \prime S}$ & $52^{\circ} 53^{\prime} 02^{\prime \prime W}$ & 12.v.2014 & - & - & TA22 & - & UFMG \\
\hline E. pseudocingulata & Brazil & Goiás & $18^{\circ} 15^{\prime} 38^{\prime \prime S}$ & $52^{\circ} 53^{\prime} 02^{\prime \prime} \mathrm{W}$ & 4.x.2014 & EP025 & EP025 & - & - & UFMG \\
\hline
\end{tabular}


Table 1. Voucher ID, locality data and institutional repositories of the specimens used in the present study (continued).

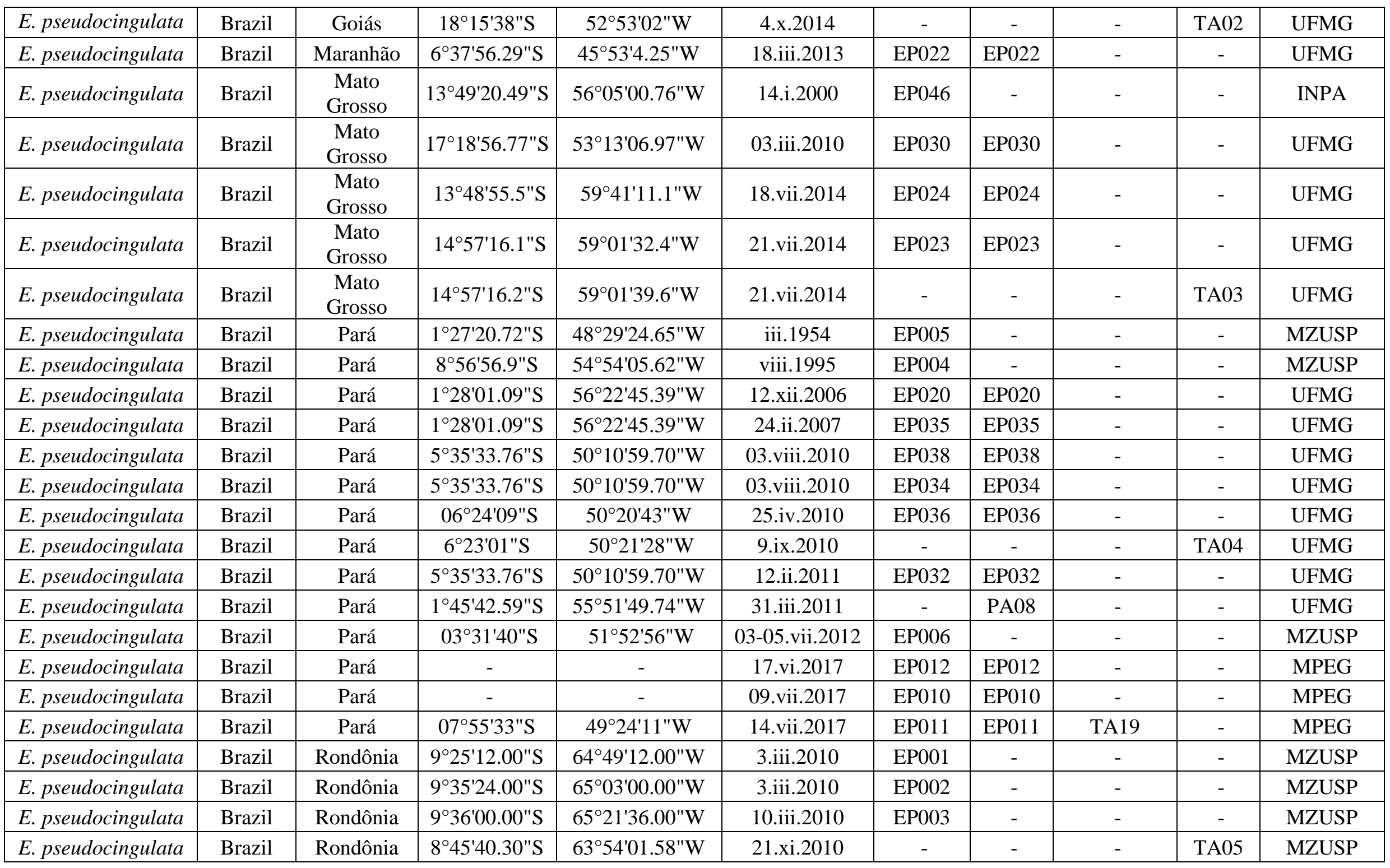


Table 1. Voucher ID, locality data and institutional repositories of the specimens used in the present study (continued).

\begin{tabular}{|c|c|c|c|c|c|c|c|c|c|c|}
\hline E. pseudocingulata & Brazil & Rondônia & $8^{\circ} 49^{\prime} 33.94 " \mathrm{~S}$ & $63^{\circ} 57^{\prime} 47.16^{\prime \prime} \mathrm{W}$ & $22 . x i .2011$ & EP026 & EP026 & - & - & UFMG \\
\hline E. pseudocingulata & Brazil & Rondônia & $8^{\circ} 49^{\prime} 33.94 " \mathrm{~S}$ & $63^{\circ} 57^{\prime} 47.16^{\prime \prime} \mathrm{W}$ & 10.1.2012 & EP027 & EP027 & - & - & UFMG \\
\hline E. pseudocingulata & Brazil & Roraima & $3^{\circ} 25^{\prime} 00.00^{\prime \prime} \mathrm{N}$ & $61^{\circ} 40^{\prime} 00.00^{\prime \prime} \mathrm{W}$ & 20-30.iii.1987 & EP045 & - & - & - & INPA \\
\hline E. pseudocingulata & Brazil & Roraima & $3^{\circ} 45^{\prime} 19.52^{\prime \prime} \mathrm{N}$ & $61^{\circ} 43^{\prime} 03.22^{\prime \prime} \mathrm{W}$ & 14.vii.2009 & EP048 & - & - & - & INPA \\
\hline E. pseudocingulata & Brazil & Roraima & $03^{\circ} 46^{\prime} 56.2^{\prime \prime} \mathrm{N}$ & $61^{\circ} 43^{\prime} 15.3^{\prime \prime W}$ & 15.vii.2014 & EP017 & EP017 & - & - & UFMG \\
\hline E. pseudocingulata & Brazil & Roraima & $03^{\circ} 46^{\prime} 56.2^{\prime \prime} \mathrm{N}$ & $61^{\circ} 43^{\prime} 15.3^{\prime \prime} \mathrm{W}$ & 15.vii.2014 & EP018 & EP018 & - & - & UFMG \\
\hline E. pseudocingulata & Brazil & Roraima & $02^{\circ} 52^{\prime} 38.4^{\prime \prime} \mathrm{N}$ & $60^{\circ} 43^{\prime} 13.1^{\prime \prime} \mathrm{W}$ & 24.vii.2014 & EP031 & EP031 & - & - & UFMG \\
\hline E. pseudocingulata & Brazil & Roraima & $2^{\circ} 44 ' 47.93 " \mathrm{~N}$ & $62^{\circ} 11^{\prime} 46.40^{\prime \prime} \mathrm{W}$ & 18-21.xii.2017 & EP049 & - & - & - & INPA \\
\hline E. pseudocingulata & $\begin{array}{l}\text { French } \\
\text { Guiana }\end{array}$ & Cayenne & $4^{\circ} 55^{\prime} 20.71 " \mathrm{~N}$ & $52^{\circ} 18^{\prime} 48.43^{\prime \prime W}$ & 1.i.2004 & - & - & DWR0071 & - & $\begin{array}{c}\text { BoldSystem } \\
\text { s }\end{array}$ \\
\hline E. pseudocingulata & Peru & $\begin{array}{c}\text { Madre de } \\
\text { Dios }\end{array}$ & $12^{\circ} 28^{\prime} 50.11 " \mathrm{~S}$ & $70^{\circ} 20^{\prime} 32.21 " \mathrm{~W}$ & 23.vii.2010 & - & - & - & ML510 & $\begin{array}{c}\text { López-Uribe } \\
\text { lab. }\end{array}$ \\
\hline E. sororia & Colômbia & Chocó & $5^{\circ} 29^{\prime} 58.86^{\prime \prime} \mathrm{N}$ & $76^{\circ} 32^{\prime} 33.35^{\prime \prime} \mathrm{W}$ & 30.vii.2006 & - & - & - & ML029 & $\begin{array}{c}\text { López-Uribe } \\
\text { lab. }\end{array}$ \\
\hline
\end{tabular}




\subsection{Morphometric data}

We use two different approaches for the geometric morphometric analysis, one considering the species and the other considering the geographical origins (Amazon Forest and Atlantic Forest). The specimen from the State of Goiás (Brazil) was removed in the last analysis, since it was collected in a Savannah area. Specimens were photographed using a Leica DFC 295® camera attached to a stereomicroscope Leica M205C®, taking all precautions to avoid possible distortions. From each specimen the right forewing was separated from the body at the base of the radial vein with the help of forceps and fixed between glass microscopy slides to keep it flat. The heads were always photographed in frontal view. The photographs were transformed into TPS files using the software TpsUtil 1.60 (Rohlf, 2013). Landmarks were set using the software tpsDig version 2.26 (Rohlf, 2006). We selected 18 homologous landmarks on the vein intersections for the wing analysis and 10 landmarks for the head (Figure 2).

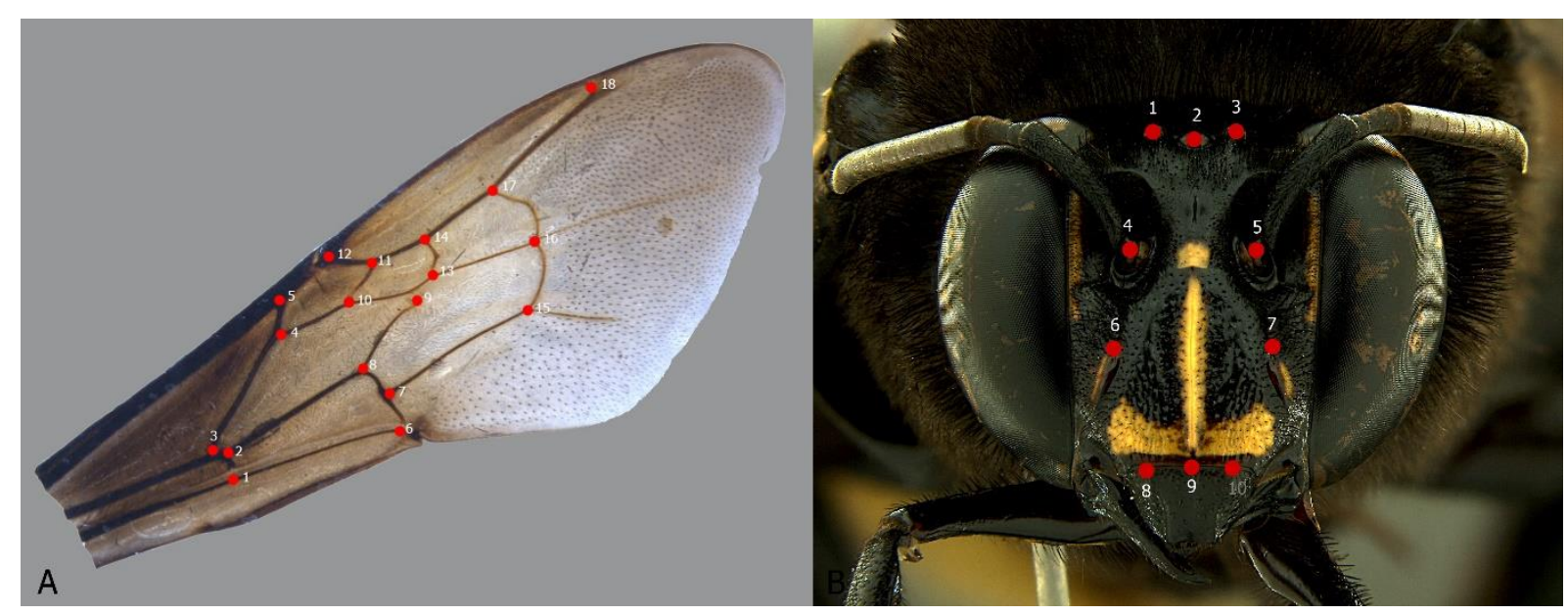

Figure 2. Landmarks used in the geometric morphometric analyses of (A) forewings and (B) head in frontal view of males of Eulaema cingulata and Eulaema pseudocingulata.

For comparison of overall wing and head sizes between species and geographical groups, we extracted the centroid size information obtained from the Procrustes Analysis. A regression analysis was performed to quantify the effect of allometry in our data. Afterwards, we removed this allometric effect in order to quantify independently the shape variation. Shape variables were obtained by performing a generalized Procrustes analysis which eliminates the effects of orientation, position and size by scaling all configurations to the same centroid size (Klingenberg, 2015). The resulting landmark configurations retain only shape information without effects of position, orientation and size. A Principal Component Analysis (PCA) was 
carried out using the relative Cartesian coordinates of each landmark after alignment. The shape difference between species and geographical clusters were tested using a Discriminant Analysis followed by a leave-one-out Cross-validation test (Lachenbruch, 1967). All analyses were performed using the software MorphoJ (Klingenberg, 2011). Additionally, we used the percentages of correct classification to evaluate the discriminatory power of wing and head shapes.

\subsection{Amplification and sequencing of COI}

Mitochondrial cytochrome oxidase I (COI) was amplified using the commonly employed barcode fragment ( 700 bp) for species delimitation: primers LCO (5'GGTCAACAAATCATAAAGATATTGG-3') and HCO (5'TAAACTTCAGGGTGACCAAAAAATCA-3') (Folmer et al., 1994). Polymerase Chain Reaction (PCR) amplifications were performed in a final volume of $17 \mu$ including $0.13 \mu \mathrm{L}$ of Taq Polymerase, $2 \mu \mathrm{L}$ of genomic template, $1.3 \mu \mathrm{L}$ of each primer, $3.4 \mu \mathrm{L}$ of dNTPs $(10 \mathrm{mM})$, $0.85 \mu \mathrm{L}$ of $\mathrm{MgCl}, 1.7 \mu \mathrm{L}$ of $10 \mathrm{x}$ Qiagen Buffer; the remaining volume $(6.32 \mu \mathrm{L})$ was filled with purified water. Amplification were performed with an initial step of three minutes at $94^{\circ} \mathrm{C}$, followed by 40 cycles of 30 seconds at $94^{\circ} \mathrm{C}, 30$ seconds for annealing at $50^{\circ} \mathrm{C}$ and $1 \mathrm{~min}$ at 72 ${ }^{\circ} \mathrm{C}$. After 40 cycles, a final step was performed at $72^{\circ} \mathrm{C}$ for 10 minutes.

All steps related to DNA extraction and amplification were performed at the Molecular Biology Laboratory of the Museu de Zoologia da Universidade de São Paulo, Brazil (MZSP). Successful amplifications were confirmed with gel electrophoresis and sent to Macrogen (Seoul, South Korea) for post-PCR clean-up and Sanger sequencing. PCR products were sequenced in two directions and sequence quality was determined by the quality scores provided by Macrogen and by visually examining the chromatograms using the software Ugene (Okonechnikov et al., 2012).

\subsection{UCE library preparation}

We used the Ultra Conserved Element (UCE) approach to phylogenomics (Faircloth et $a l ., 2012)$ to generate a genome-scale data set. This method combines the targeted enrichment of thousands of UCE loci with multiplexed next-generation sequencing. A recent published bait set specific to bees, ants and other apoid wasps was used to perform the enrichment ("hym-v2bee-ant-specific"; Grab et al., 2019). This bait set is a subset of the principal Hymenoptera bait set first reported in Branstetter et al. (2017). Each DNA sample was sheared using a Qsonica Q800R2 acoustic sonicator, with the target fragment size range being 400-600 bp (60-120 secs 
shear time, 25\% amplitude, 10-10 sec pulse). Having larger fragment sizes in the sequencing pool improves the amount of flanking DNA that gets sequenced and improves assembled contig lengths. For recently collected, high quality samples, the sonicator was run for 90-120 seconds (shearing time) at 25\% amplitude and with a 10-10 second on-off pulse. For relatively older samples that likely had more degraded DNA, we adjusted the shearing times to between 30-60 seconds. Following sonication, fragmented DNA was cleaned at $3 \mathrm{x}$ volume using a homemade SPRI-bead substitute (Rohland \& Reich, 2012). Illumina sequencing libraries were generated for each sample using Kapa Hyper Prep Kits and custom, dual-indexing adapters (Glenn et al., 2016). Libraries were PCR amplified for 12 cycles for most samples, but for lower quality samples we increased the number to $14-16$ cycles. Each amplified library was cleaned using 1.0-1.2x SPRI beads in order to remove contaminants and to select out small fragments below $200 \mathrm{bp}$, including adapter dimer. The concentration of the final cleaned library was measured using Qubit.

Libraries were PCR amplified for 12 cycles, purified using an Ampure XP substitute (Rohland \& Reich, 2012), and quantified using Qubit. To enrich UCE loci, 10 samples were pooled at equimolar concentrations and then up to $500 \mathrm{ng}$ of each pool was enriched following the manufacturer's protocol for day 1 (MYcroarray enrichment protocol v3.02) and the standard UCE protocol for day 2 (enrichment protocol v1.5 available at ultraconserved.org). For each enrichment, the custom bait set was diluted 1:4 (1 $\mu \mathrm{L}$ bait, $4 \mu \mathrm{L} \mathrm{H} 2 \mathrm{O})$ and we performed the enrichment incubation at $65^{\circ} \mathrm{C}$ for 24 hours using strip tubes and a PCR thermal cycler. For the second day of enrichment, we used $50 \mathrm{uL}$ of streptavidin beads per sample and performed onbead PCR following the three heated $\left(65^{\circ} \mathrm{C}\right)$ wash steps. The enriched pools were amplified for 18 cycles and the resulting products were cleaned with SPRI beads at $1 \mathrm{x}$ volume. Following enrichment, each enrichment pool was quantified using qPCR and pooled together into a final sequencing pool at equimolar concentrations. Sequencing pools were sent to either the University of Utah Genomics Core for sequencing on an Illumina HiSeq 2500 (2x125, v4 chemistry), or to Novogene for sequencing on HiSeq X.

\subsection{Bioinformatics}

COI sequences were aligned using multiple sequence alignment online tool MAFFT (Katoh et al., 2019) and manually edited using the software Ugene (Okonechnikov et al., 2012). For the phylogenomic analysis, we cleaned the reads for adapter contamination and low-quality bases using Illumiprocessor (Faircloth, 2013) incorporated in the software Trimmomatic (Bolger et al., 2014). We assembled reads de novo for each individual using Spades (Bankevich 
et al., 2012). To identify UCE regions from the bulk of assembled contigs and to remove paralogs we employed the HymV2-bee-ant UCE bait files from Branstetter (2017). We aligned individually all the loci using MAFFT (Katoh \& Standley, 2013), and we trimmed resulting alignments using GBLOCKS (Castresana, 2000; Tavalera \& Castresana, 2007). We removed loci that had data for fewer than $75 \%$ of taxa and generated a concatenated matrix from the resulting alignment set.

\subsection{Molecular approaches for species phylogeny and delimitation}

Estimation of the genetic distance among specimens was calculated by MEGA-X (Kumar et al., 2018) with 10.000 bootstraps. We inferred phylogenetic trees using Maximum Likelihood (ML), Bayesian Inference (BI) and Species Tree (ST) methods. Maximum likelihood analyses were inferred using two different strategies: single concatenated alignment and partitioned based on the best-fitting partitioning scheme. We test these two phylogenetic model-based methods since concatenated-based methods infer phylogenies that assume that all genes have similar histories; on the other hand, when the dataset is partitioned, different models of evolution are applied to individual loci (Young \& Gillung, 2020). The best-fitting partitioning scheme was obtained using Sliding-Window Site Characteristics (SWSC). This approach divides each UCE into three data blocks, corresponding to the right flank, core and left flank (Tagliacolo \& Lanfear, 2018). After running the SWSC-EN algorithm, the resulting data subsets were analyzed using PartitionFinder2 (Lanfear et al., 2017). For this analysis, we used the rclusterf algorithm, AICc model selection criterion and GTR+G model of sequence evolution. The best partitioning scheme grouped loci having the same substitution model to be used in the subsequent analyses. We used the likelihood-based program Iq-Tree v.1.6.12 (Nguyen et al., 2015) for both partitioning schemes. The substitution model of the concatenated alignment (TVM+F+R2) was obtained with the ModelFinder implemented in the Iq-Tree software (Kalyaanamoorthy et al., 2017). To assess branch support, we performed 1,000 replicates of the ultrafast bootstrap approximation (UFBoot; Hoang et al., 2018). Additionally, 1,000 replicates of the branch-based Shimodaira-Hasegawa approximate likelihood-rate test (SH-aLRT; Guindon et al., 2010) were conducted using the command '-alrt'. Only clades with support values of UFBoot $\geq 0.95$ and SH-aLRT $\geq 0.80$ were considered robust.

We performed Bayesian phylogenetic analyses in MrBayes v.3.2 (Ronquist et al., 2012) using the best nucleotide model estimated by PartitionFinder2 (Lanfear et al., 2017). The Markov Chain Monte Carlo (MCMC) was run for 20 million generations sampled every 1000th generation. Twenty-five percent of the first trees were discarded as burnin. Bayesian trees were 
visualized and edited in FigTree v. 1.4.4 (Rambaut, 2016).

To account for heterogeneous gene histories that may influence phylogenetic accurate resolution, we inferred a species trees under the multi-species coalescent model using the program ASTRAL-III v.5.7.3 (Zhang et al., 2018). For this analysis, we used the set of unrooted gene trees that were generated by UFboot in Iq-Tree to the analysis of not partitioned loci. Support was assessed as local posterior probability, with $\geq 0.95$ considered robust. We also estimated a coalescent-based species tree using *Beast (Heled and Drummond, 2010) in the Beast package (Bouckert et al., 2019), which co-estimate gene trees and species trees from input sequence alignments, and assume that individuals are already correctly assigned to species although the species phylogeny is yet to be estimated (Zhang et al., 2011). We first run the command 'phyluce_align_get_informative_sites' on Phyluce to get the 100 most informative genes, from these we manually chose those that were present in all samples (resulting in 88 UCEs). The analysis was run for 100 million generations sampling every 10.000 generations under a strict clock model with a constant population model, and a Yule model as a tree prior. We used a GTR model (unlinked across loci) for the nucleotide substitution model that was provided by PartitionFinder2 (Lanfear et al., 2017). Tracer v1.7 was used to examine the convergence across runs and the ESS values of sampled parameters. We constructed a maximum clade credibility in TreeAnnotator and visualized the tree using Densitree, both included in the Beast package.

\section{RESULTS}

\subsection{Morphometric geometrics}

Regarding the variation in shape of the head, the PCA generated eight Principal Components. The first three components explained $45.28 \%, 19.16 \%$ and $12.37 \%$ of the covariance, respectively, totalizing $76.81 \%$. The discriminant analysis correctly assigned $64.4 \%$ of the specimens to their respective nominal species and $55.17 \%$ to their geographic groups in the cross-validation test. The PCA plot showed superimposition among both groups (Figure 3). Regarding size, the multivariate regression analysis showed that $32.28 \%$ of the shape is explained by the allometric effect of the size, with $\mathrm{P}<0.0001$. Nonetheless, even after removing this allometric effect, the groups remained undifferentiated. The comparison between heads centroid sizes showed that there are no significant differences between both nominal species and geographical groups (Figure 3). 
The PCA of wings generated 30 Principal Components, of which the first three explained $39.23 \%$ of the variation. The first three components explained the $14.59 \%, 13.89 \%$ and $10.75 \%$. of the variance, respectively. The cross-validation test correctly located $67 \%$ of the nominal species and $61.62 \%$ of the geographical groups. A superimposition in PCA plots was also observed in the results of wing's analysis (Figure 4). The regression analysis showed a lower effect of size on shape compared to the ones of the head analysis, 3.59\% ( $\mathrm{P}=0.007)$ of variation is explained by the size.
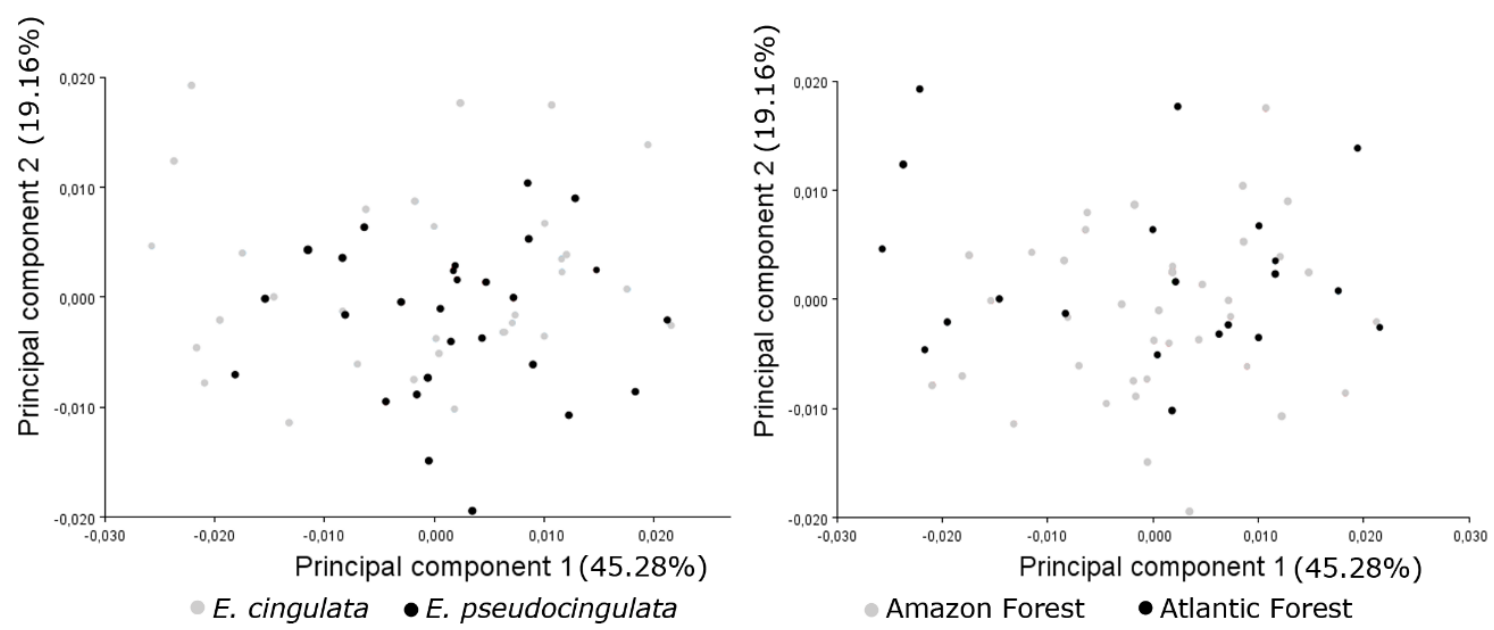

Figure 3. Shape variation of male heads grouped by: A. Different nominal species, and B. Different geographic groups; by PC 1 and PC 2. The percentage explained by each Principal Components is in parenthesis. The negative and positive extremes of both PC1 and PC2 are shown below and besides of the graph (Factor scale: left -0.03 , right 0.03 ).
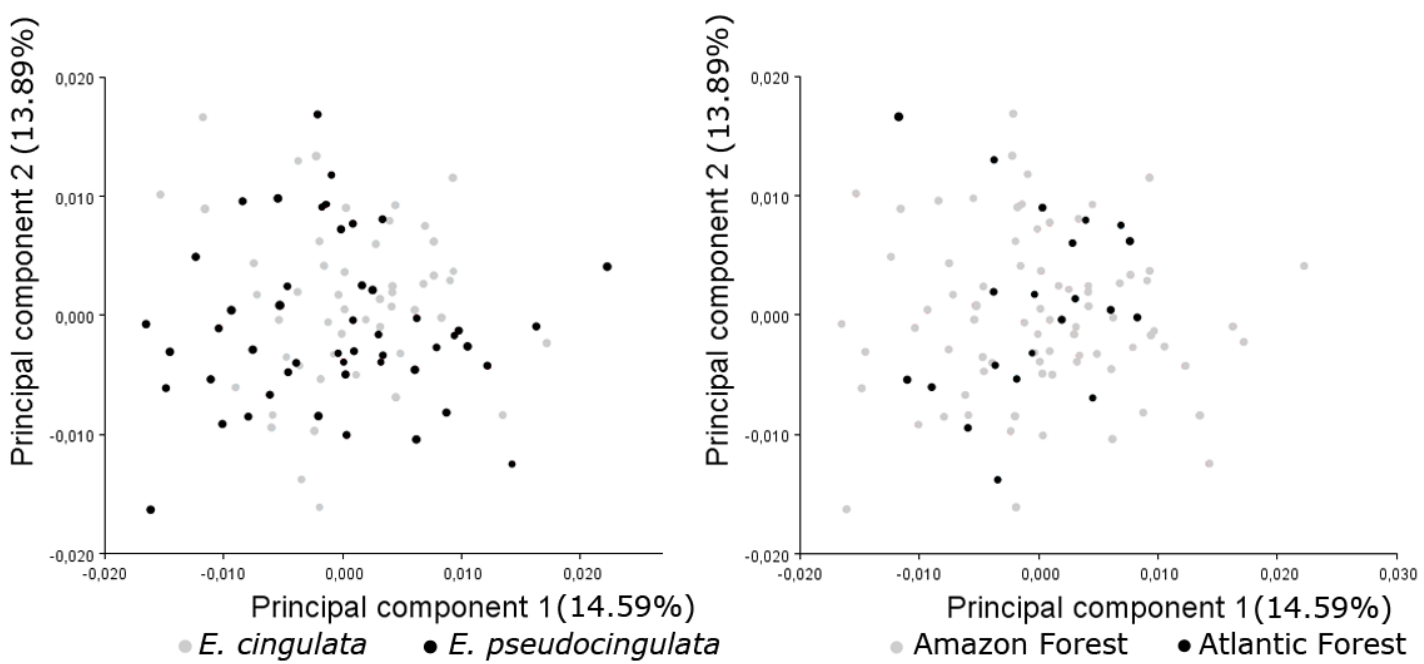

Figure 4. Shape variation of male wings grouped by: A. Different nominal species, and B. 
Different geographic groups; by PC 1 and PC 2. The percentage explained by each Principal Components is in parenthesis. The negative and positive extremes of both PC1 and PC2 are shown below and beside of the graph (Factor scale: left -0.03 , right 0.03 ).

\subsection{Genetic distance}

The average pairwise genetic p-distance within $E$. cingulata was $1.3 \%$ and within $E$. pseudocingulata was $0.7 \%$, while between the two species was $0.95 \%$. This amount of genetic differentiation in mitochondrial DNA indicates no significant difference supporting the presence of two evolutionarily independent units. Additionally, the average pairwise genetic distances among E. cingulata and the other analyzed nominal species were between $5.6 \%$ and 11.2\%, and among E. pseudocingulata and the others were between $5.32 \%$ and $11.35 \%$. All genetic distances results within and between nominal species are summarized in Table 2.

Table 2. Average pairwise genetic p-distance between nominal species of Eulaema.

\begin{tabular}{|l|c|c|}
\cline { 2 - 3 } \multicolumn{1}{c|}{} & Eulaema cingulata & Eulaema pseudocingulata \\
\hline Eulaema cingulata & $1.3 \% *$ & $0.95 \%$ \\
\hline Eulaema pseudocingulata & $0.95 \%$ & $0.7 \% *$ \\
\hline Eulaema mocsaryi & $5.6 \%$ & $5.32 \%$ \\
\hline Eulaema nigrita & $9.3 \%$ & $9.06 \%$ \\
\hline Eulaema meriana & $11.2 \%$ & $11.35 \%$ \\
\hline
\end{tabular}

*Average pairwise genetic p-distance within each species.

\subsection{Phylogenetic relationships}

COI sequences were aligned resulting in an alignment of $655 \mathrm{bp}$. JModel Test identified GTR $+\mathrm{I}$ as the best nucleotide substitution model. The consensus tree obtained from MrBayes shows that the COI fragments group E. cingulata and E. pseudocingulata into one highly supported clade sister of E. mocsaryi (Figure 5).

Analysis of the UCE data comprises a total of 21,470,135 reads with an average of $1,431,342$ reads per sample (range $=513,594-2,585,935$ ). These reads were assembled into an average of 186,700 contigs per sample (range $=34,358-441,504$ ), having an average length of $167 \mathrm{bp}$. An average (per sample) of 2,217 of those contigs matched the UCE loci from the target capture probes used. Following the alignment, trimming, and filtering of the UCE contigs, our UCE matrix consisted of 2,180 loci and 1,509,760 bp of sequence data, of which 33,722 bp were informative. The average length of UCE-matching contigs was about $692 \mathrm{bp}$ $($ range $=229-1,831)$. 
The UCEs phylogenetic hypothesis recovered from both partitioned and concatenated schemes used for maximum likelihood inference showed identical topologies, with most nodes receiving high supports (Figures 6.A,B). The group including all specimens of Eulaema cingulata and E. pseudocingulata was found to be monophyletic with maximum support. The difference between the trees is in the phylogenetic position of Eulaema pseudocingulata TA03 and TA04 within a small pseudocingulata's clade. The only individual from the Atlantic Forest (Eulaema cingulata TA09) was recovered with maximum support as sister to the individual from Mato Grosso (Eulaema cingulata TA07), a state in the Amazon Forest. The ASTRAL and Star Beast species tree had highly support values and both recovered the same topology (Figure $6 C)$.

Even though the COI data included different samples from the UCE data, both methods presented very similar topologies with no significant differences. Both trees showed one clade with the two species included and intermixed with high statistical support. The internal topology resolution show a low divergence levels between these evolutionary units. As we can see in figures 5 and 6 , the node corresponding to the species studied showed no separation between nominal species.

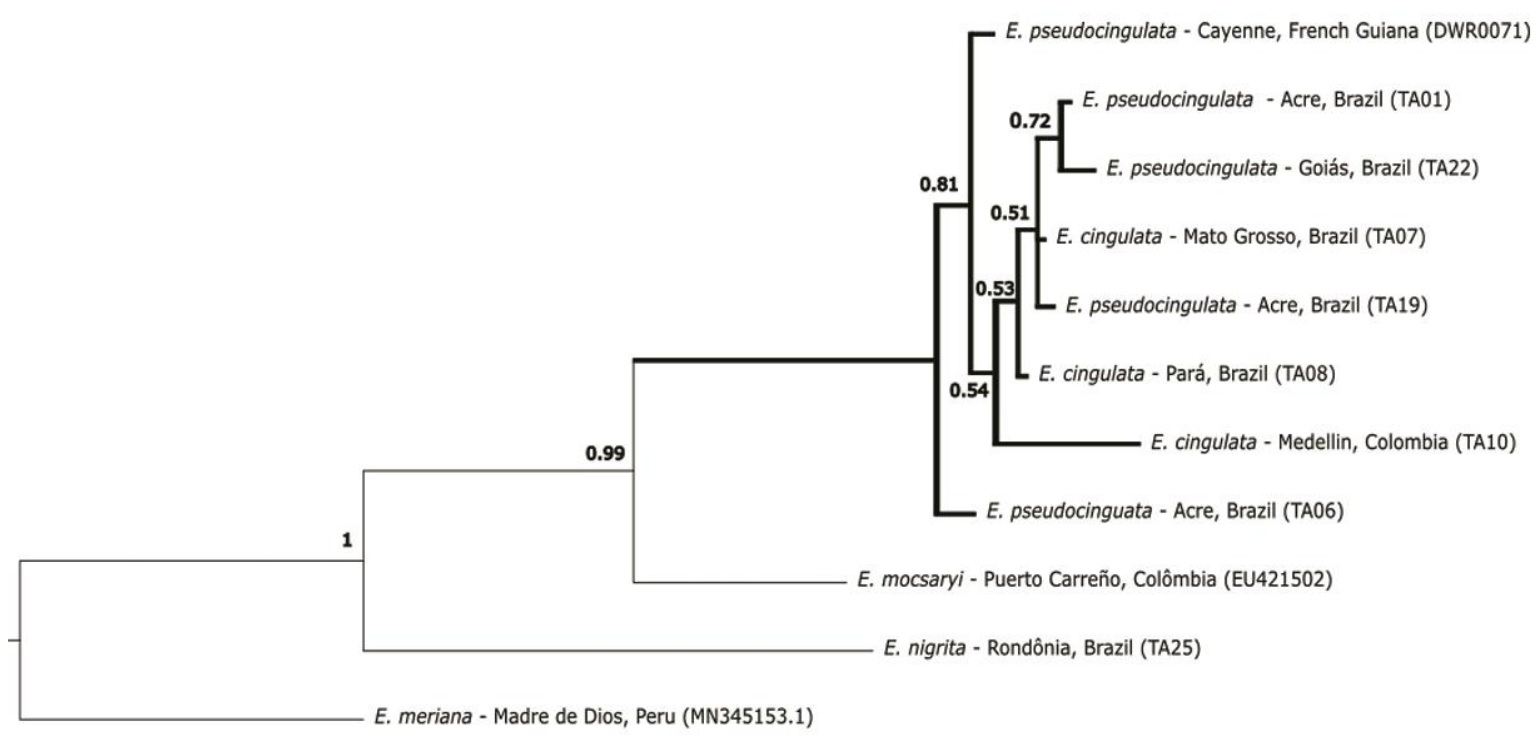

Figure 5: Consensus tree resulting from a Bayesian analysis of molecular data from the gene COI for species of the bee genus Eulaema. Posterior probability support is indicated on branches. The codes in front of each analyzed individual correspond to its geographical distribution indicated in Table 1. 

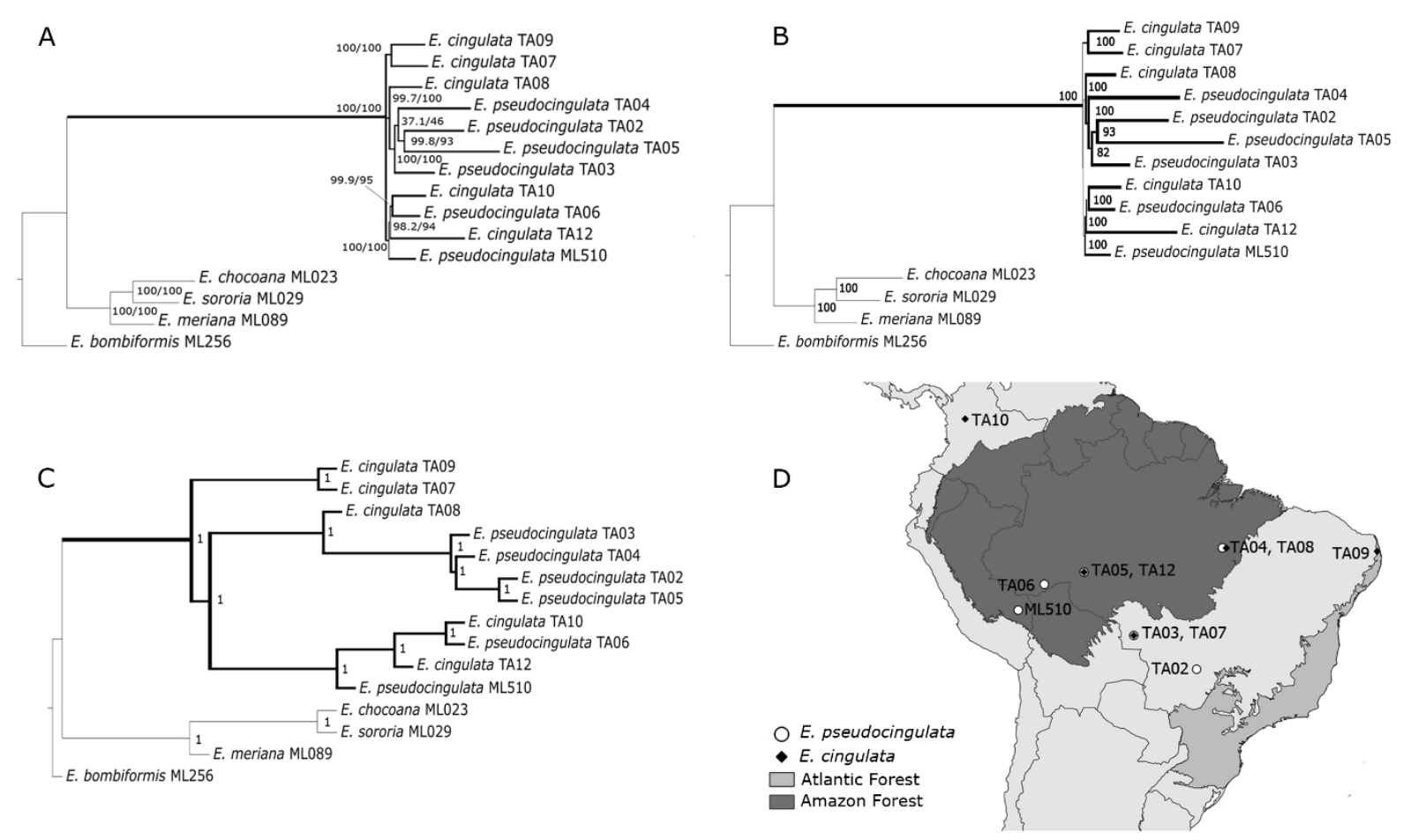

Figure 6. Phylogenetic trees for the nominal Eulaema cingulata and E. pseudocingulata obtained with UCEs. A. Maximum likelihood phylogenetic tree obtained with concatenated dataset of UCEs in Iq-Tree. Numbers on nodes correspond to Ultrafast bootstrap (1000 replicates) and SH-like, respectively. B. Maximum likelihood phylogenetic tree obtained with partitioned dataset of UCEs in Iq-Tree. Numbers on nodes correspond to Ultrafast bootstrap (1000 replicates). C. Species delimitation analyses based on multispecies-coalescent model obtained with ASTRAL and StarBeast. Number on nodes corresponds to posterior probabilities. D. Map showing the distribution of the analyzed material of ingroup.

\section{DISCUSSION}

A species concept that is in accordance with integrative taxonomy is the General Lineage Concept (GLC), which only required property is that species evolve separately from other lineages (De Queiroz, 2007). Other criteria such as diagnosability, monophyly, or reproductive isolation can be used to recognize separately evolving lineages. The taxonomy of Eulaema cingulata and E. pseudocingulata has long remained controversial. Based on a multidisciplinary approach, we investigated if these species could be recognized as different taxonomic units. Our morphometric results showed no clustering for the analyzed species neither for different geographic origins, nor among intra or interspecific populations. Geometric 
morphometrics of the forewings have been proved as a powerful technique to discriminate bees' species (Francoy et al., 2012; Combey et al., 2013), subspecies (Oleksa \& Tofilski, 2014; Silva et al., 2015), cryptic species (Francisco et al., 2008, Hurtado-Burillo et al., 2016) and geographical ecotypes (Francoy et al., 2011; Grassi-Sella et al., 2018, Carneiro et al., 2019). Using both landmarks and outlined-based methodologies, Francoy et al. (2012) showed that the use of this approach to discriminate Euglossa species was more effective than studies using allozymes and restriction patterns of mitochondrial genes. Quezada-Euán et al. (2015) identified differences in wing shape of the two different morphs of Euglossa viridissima that had been otherwise identified only by the number of mandibular teeth. The morphometrics study of wings of Euglossa annectans Dressler, 1982 and Euglossa truncata Rebêlo \& Moure, 1995 sampled in the Atlantic Forest and Savannah in Brazil, reported the high morphological similarity between populations albeit the comparatively high dispersal of these bees (GrassiSella et al., 2018). We applied the study to the heads in order to assess and compare its power to distinguish among closely related species or forms. The results were congruent with the results obtained with the study of the forewings. Despite being less used than the wings, the use of landmarks on the head has been also informative to recognize intercastes in honeybees (Souza et al., 2015), and to discriminate the morphologically indistinguishable females of the Psychodopygus complex (Diptera) (Godoy et al., 2018).

The level of morphological differentiation among recently divergent lineages is sometimes insufficient to recognize species and the use of DNA data has been a useful tool to test the presence of genetically distinct groups of individuals for which one could not find discrete morphological differences. Based on our data, the pair-wise sequence divergences within and between the two species were below 3\%, which according to Hebert et al.(2003) is a result compatible with the expected variation within a single species. When compared with recognized species, a 'barcode gap' is verified with interspecific distances always larger than 3\% (Table 2). Dick et al. (2004) found mtDNA divergences within Euglossini species consistently low, with divergences among populations separated by the Andes averaged 1.1\%, and $0.4 \%$ of mean divergence among cross-Amazon samples (collection sites cover $3.000 \mathrm{~km}$ ). These findings suggest high levels of long-distance gene flow between populations. RochaFilho et al. (2013) observed a comparatively high dispersal ability in Eulaema cingulata through analyses on genetic differentiation between mainland and island populations. LópezUribe et al. 2014 also found low values of mitochondrial nucleotide divergence between populations of Euglossini, showing a minimum value of $0.39 \%$ in E. cingulata among the studied species. According to the authors, the low sequence divergence between populations of 
Eulaema cingulata was probably due to the recent origin of this species. The low sequence divergence between individuals of E. cingulata found in our study corroborates previous reports, characterizing this bee as displaying large populations with high gene flow.

DNA barcodes are increasingly becoming a standard tool used by taxonomists and its association with morphological characters has proven to help species discrimination in several groups of bees. Although this method has been criticized by some authors (Rubinoff et al., 2006; Wheeler, 2008), it gives additional support to the recognition of species when considered along with other data sources (Padial \& De La Riva, 2007). Our phylogenetic analysis yielded an informative tree, providing strong support for the hypothesis that E. cingulata and E. pseudocingulata belong to the same evolutionary unit. A phylogenetic study using morphological characters recovered these two morphs occupying different species groups within the subgenera Apeulaema (Oliveira 2006). The later author found that $E$. pseudocingulata belongs to the group 'peruviana', phylogenetically related to $E$. polychroma while E. cingulata belongs to the group 'nigrita' with E. nigrita, E. mocsaryi and E. boliviensis. The first group can be recognized by the lateral projection of the eighth sternum short and rounded, a character that occurs also in the subgenus Eulaema (Eulaema). This suggest that this character is homoplastic and, consequently, undermines the decision of recognizing species groups within the subgenus Apeulaema. The recent revision of the subgeneric classification in Eulaema recognized E. pseudocingulata as not closely related to $E$. polychroma but as a member of the same species group that E. cingulata (Melo, 2014).

The topology obtained with genomic data supports the monophyly and recognition of the clade that comprises E. cingulata and E. pseudocingulata as previously recognized by COI data. This result corroborate UCEs as another useful tool that provides sufficient variation at shallow time scales to enable species discrimination, adding the utility of these markers for species delimitation studies as observed in other studies (e. g. Smith et al., 2013; Pie et al., 2019; Gueuning et al., 2020). Combined phylogenetic and population genetic approaches were effective to investigate boundaries between complexes of wild European bees suspect to harbor cryptic diversity, mitochondrial introgression, or mitochondrial paraphyly (Gueuning et al., 2020). Using COI and UCEs with multispecies coalescent method (BPP), Gueuning et al. 2020 noticed that UCEs provided robust species hypotheses and can outperform COI in species delimitation. The adoption of delimitation methods based on the multispecies coalescent model has been criticized by Sukumaran and Knowles (2017), who argued that these methods tend to delimit population structure instead of species. The authors' concern was raised by the possibility of taxonomic inflation if species are described based only on molecular data. 
However, most studies using genetic data in species delimitation also incorporate other sources of data and very few studies have been based only on genetic data. The use of species delimitation approaches may increase reliance if different methods offer congruent results within a given dataset.

Herein we conclude that E. pseudocingulata does not correspond to an independent evolutionary lineage from E. cingulata. Yet, the difference in the shape of the mid tibia velvet area, proposed as the diagnostic character between the morphs, can be indeed interpreted as a variation condition. It can be narrower and farther from the rear edge in some morphs occurring in the Amazon Forest or wider and closer to the rear edge, in morphs occurring throughout the species distribution. Variation is broadly present in bees. Bumble bees, for example, are known for presenting high variability in its coloration pattern (e. g. Carolan et al., 2012; Huang et al., 2015; Koch et al., 2018). Color variation in Eucerini bees was reported by Grando et al., (2018) that found two distinct color patterns in sympatric populations of Melissodes nigroaenea in Brazil. Variation in color and shape was observed in Augochlora amphitrite by Lepeco \& Gonçalves (2018); using morphometric analyses and studying the male genital capsules; the authors did not find any character that could support the recognition of distinct color morphs and macrocephalic females as different species. A study of different color morphs of Euglossa species from the Atlantic Forest that were considered as distinct species, received no phylogenetic support, nor enough genetic distance to justify the recognition of the color morphs as different species (Ferrari \& Melo, 2014).

We do not know the mechanisms responsible for maintaining this variation in individuals of Eulaema cingulata in the Amazon Forest. A similar case was found in a study of two sympatric Euglossa species - E. viridissima and E. dilemma. These are sister species that can be differentiated by the number of mandibular teeth; E. viridissima possess two teeth while E. dilemma has three with some males of E. viridissima expressing a third tooth. These species are distinguished by chemical characters (cuticular hydrocarbons found in the hind tibia) as well as by highly variable DNA markers (microsatellites and SNPs), but show no systematic variation amongst the morphs of $E$. viridissima, confirming that it is in fact a variable species (Eltz et al., 2011; Pokorny et al., 2014; Quezada-Euán et al., 2015). It is difficult to suggest a mechanism for the maintenance of the two morphs in males of E. cingulata. However, it is important to understand how genetic diversity is maintained within these populations, and in this sense, further investigations are necessary focusing on the factors that lead to these variations. 


\section{CONCLUSIONS}

We evaluated here possible species limits of Eulaema cingulata and E. pseudocingulata by integrating multiple independent datasets: geometric morphometrics, mitochondrial DNA, and phylogenomics using ultraconserved elements. All obtained results were congruent regardless of the used methods, showing no separation between morphs that were previously recognized as different species. Our results also suggest that the morphology of the mid tibia of E. pseudocingulata, proposed as the diagnostic character between the morphs, is a variable condition of some individuals of E. cingulata from the Amazon Forest. Besides the variation in the mid leg, there is also a color variation in some morphs, which causes are yet unknown. Orchid bees are important pollinators in Neotropical forests. In addition to being widely used in environmental quality studies, they have become a good model for evolutionary genetics studies. However, as shown here, there is still the need to ameliorate our knowledge of species delimitation in these bees, even in some of the most ordinary and widespread species in the Neotropical Region.

\section{REFERENCES}

Austen, G.E.; Bindemann, M.; Griffiths, R.A. \& Roberts, D.L. 2016. Species identification by experts and non-experts: comparing images from field guides. Scientific Reports, 6(33634): 1-7. doi: 10.1038/srep33634

Bankevich, A.; Nurk, S.; Antipov, D.; Gurevich, A.A.; Dvorkin, M.; Kulikov, A.S.; Lesin, V.M.; Nikolenko, S.I.; Pham, S.; Prjibelski, A.D.; Pyshkin, A.V.; Sirotkin, A.V.; Vyahhi, N.; Tesler, G.; Alekseyev, M.A. \& Pevzner, P.A. 2012. SPAdes: a new genome assembly algorithm and its applications to single-cell sequencing. Journal of Computational Biology, 19(5): 455-477. doi: 10.1089/cmb.2012.0021.

Bejerano, G.; Pheasant, M.; Makunin, I.; Stephen, S.; Kent, W.J.; Mattick, J.S. \& Haussler, D. 2004. Ultraconserved elements in the human genome. Science, Washington, 304(5675): 1321-1325. doi: 10.1126/science.1098119.

Blaimer, B.B.; Lloyd, M.W.; Guillory, W.X. \& Brady, S.G. 2016. Sequence capture and phylogenetic utility of genomic ultraconserved elements obtained from pinned insect specimens. PLoS ONE, 11: e0161531. doi:10.1371/journal.pone.0161531.

Blaxter, M.L. 2004. The promise of a DNA taxonomy. Philosophical Transactions of the 
Royal Society B, 359(1444): 669-679.

Bolger, A.M.; Lohse, M. \& Usadel, B. 2014. Trimmomatic: a flexible trimmer for Illumina sequence data. Bioinformatics, 30(15): 2114-2120. doi: 10.1093/bioinformatics/btu170.

Bouckaert, R.R.; Vaughan, T.G.; Barido-Sottani, J.; Duchene, S. Fourment, M.; Gavryushina, A.; Heled, J.; Jones, G.; Kühnert, D.; Maio, N.; Matschiner, M.; Mendes, F.K.; Müller, N.F.; Ogilvie, H.A.; Plessis, L.; Popinga, A; Rambaut, A.; Rasmussen, D.; Siveroni, I.; Suchard, M.A.; Wu, C-H.; Xie, D.; Zhang, C.; Stadler, T. \& Drummond, A.J. 2019. BEAST 2.5: An advanced software platform for Bayesian evolutionary analysis. PLoS Computational Biology, 15: e1006650. doi: 10.1371/journal.pcbi.1006650.

Branstetter, M.G.; Longino, J.T.; Ward, P. S. \& Faircloth, B.C. 2017. Enriching the ant tree of life: enhanced UCE bait set for genome-scale phylogenetics of ants and other Hymenoptera. Methods in Ecology and Evolution 8(6): 768-776. doi: 10.1111/2041210x.12742.

Cardoso, P.; Erwin, T.L.; Borges, P.A.V. \& New, T.R. 2011. The seven impediments in invertebrate conservation and how to overcome them. Biological Conservation, 144(11): 2647-2655. doi: 10.1016/j.biocon.2011.07.024.

Carneiro, L.S.; Aguiar, C.M.L.; Aguiar, W.M; Aniceto, E.S.; Nunes, L.A. \& Ferreira, V.S. 2019. Morphometric variability among populations of Euglossa cordata (Hymenoptera: Apidae: Euglossini) from different phytophysiognomies. Sociobiology, 66(4): 575-581. doi: 10.13102/sociobiology.v66i4.4675.

Carolan, J.C.; Murray, T.E.; Fitzpatrick, Ú.; Crossley, J.; Schmidt, H.; Cederberg, B.; McNally, L.; Paxton, R.J.; Williams, P.H. \& Brown, M.J.F. 2012. Color patterns do not diagnose species: quantitative evaluation of a DNA barcode cryptic bumblebee complex. PLoS ONE, 7: e29251. doi: 10.1371/journal.pone.0029251.

Carstens, B.C. \& Dewey, T.A. 2010. Species delimitation using a combined coalescent and information-theoretic approach: an example from North American Myotis bats. Systematic Biology, 59(4): 400-414. doi: 10.1093/sysbio/syq024.

Carstens, B.C. \& Satler, J.D. 2013. The carnivorous plant described as Sarracenia alata contains two cryptic species. Biological Journal of the Linnean Society, 109(4):737746. doi: 10.1111/bij.12093.

Castresana, J. 2000. Selection of conserved blocks from multiple alignments for their use in 
phylogenetic analysis. Molecular Biology and Evolution, 17(4): 540-552. doi: 10.1093/oxfordjournals.molbev.a026334.

Cavalcante, M.C.; Oliveira, F.F.; Maués, M.M. \& Freitas, B.M. 2012. Pollination requirements and the foraging behavior of potential pollinators of cultivated Brazil nut (Bertholletia excelsa Bonpl.). Trees in Central Amazon Forest. Psyche, Cambridge, 2012(978019): 19. doi: 10.1155/2012/978019.

Combey, R.; Teixeira, J.S.G.; Bonatti, V.; Kapwong, P. \& Francoy, T.M. 2013. Geometric morphometrics reveals morphological differentiation within four African stingless bee species. Annals of Biological Research, 4:95-105.

Crawford, N.G.; Faircloth, B.C.; McCormack, J.E.; Brumfield, R.T.; Winker, K. \& Glenn, T.C. 2012. More than 1000 ultraconserved elements provide evidence that turtles are the sister group of archosaurs. Biology Letters, 8(5): 783-756. doi: 10.1098/rsbl.2012.0331.

Crawford, N.G.; Parham, J.F.; Sellas, A.B.; Faircloth, B.C.; Glenn, T.C.; Papenfuss, T.J.; Henderson, J.B.; Hansen. M.H. \& Simison, W.B. 2015. A phylogenomic analysis of turtles. Molecular Phylogenetics and Evolution, 83: 250-257. doi: 10.1016/j.ympev.2014.10.021.

Dayrat, B. 2005. Towards integrative taxonomy. Biological Journal of the Linnean Society, 85(3): 407-415. doi: 10.1111/j.1095-8312.2005.00503.x.

DeSalle, R.; Egan, M.G. \& Siddall, M. 2005. The unholy trinity: taxonomy, species delimitation and DNA barcoding. Philosophical Transactions of the Royal Society B, 360: 1905-1916. doi: $10.1098 /$ rstb.2005.1722.

De Queiroz, K. 2007. Species concepts and species delimitation. Systematic Biology, 56(6):879-886. doi: 10.1080/10635150701701083.

Dick, C.W.; Roubik, D.W; Gruber, K.F. \& Bermingham, E. 2004. Long-distance gene flow and cross-Andean dispersal of lowland rainforest bees (Apidae: Euglossini) revealed by comparative mitochondrial DNA phylogeography. Molecular Ecology, 13(12): 37753785. doi: 10.1111/j.1365-294X-2004.02374.x.

Dressler, R.L. 1982. Biology of the orchid bees (Euglossini). Annual Review of Ecology and Systematics, 13: 373-394. doi: 10.1146/annurev.es.13.110182.002105.

Eltz, T.; Fritzsch, F.; Pech, J.R.; Zimmermann, Y.; Ramírez, S.R.; Quezada-Euán, J.J.G.; Bembé, B. 2011. Characterization of the orchid bee Euglossa viridissima (Apidae: 
Euglossini) and a novel cryptic sibling species, by morphological, chemical, and genetic characters. Zoological Journal of the Linnean Society, 163(4): 1064-1076. doi: 10.1111/j.1096-3642.2011.00740.x.

Evangelista, O.; Sakakibara, Albino ; Cryan, J. R.; Urban, J. M. 2012. A phylogeny of the treehopper subfamily Heteronotinae reveals convergent pronotal traits (Hemiptera: Auchenorrhyncha: Membracidae). Systematic Entomology, 42(2): 410-428. doi: 10.1111/syen.12221.

Faircloth, B. C. 2013. Illumiprocessor: a trimmomatic wrapper for parallel adapter and quality trimming. Disponível em: https://github.com/faircloth-lab/illumiprocessor. doi: 10.6079/J9ILL.

Faircloth, B.C.; Branstetter, M.G.; White, N.D. \& Brady, S.G. 2015. Target enrichment of ultraconserved elements from arthropods provides a genomic perspective on relationships among Hymenoptera. Molecular Ecology Resources, 15(3): 489-501. doi: 10.1111/1755-0998.12328.

Faircloth, B.C.; McCormack, J.E.; Crawford, N.G.; Harvey, M.G.; Brumfield, R.T. \& Glenn, T.C. 2012. Ultraconserved elements anchor thousands of genetic markers spanning multiple evolutionary timescales. Systematic Biology, 61(5):7 17-726. doi: 10.1093/sysbio/sys004.

Ferrari, B.R. \& Melo, G.A.R. 2014. Deceiving colors: recognition of color morphs as separate species in orchid bees is not supported by molecular evidence. Apidologie, 45(5): 641652. doi: 10.1007/s13592-014-0280-7.

Folmer, O.; Black, M.; Hoeh, W.; Lutz, R. \& Vrijenhoek, R. 1994 DNA primers for amplification of mitochondrial cytochrome c oxidase subunit I from diverse metazoan invertebrates. Molecular Marine Biology and Biotechnology, 3(5): 294-299.

Francisco, F.O.; Nunes-Silva, P.; Francoy, T.M.; Wittmann, D.; Imperatriz-Fonseca, V.L.; Arias, M.C. \& Morgan, E.D. 2008 Morphometrical, biochemical and molecular tools for assessing biodiversity. An example in Plebeia remota (Holmberg, 1903) (Apidae, Meliponini). Insectes Sociaux, 55(3): 231-237. doi: 10.1007/s0004-008-0992-7.

Francoy, T.M.; Grassi, M.L.; Imperatriz-Fonseca, V.L.; May-Itzá, W.J. \& Quezada-Euán, J.J.G. 2011. Geometric morphometrics of the wing as a tool for assigning genetic lineages and geographic origin to Melipona beecheii (Hymenoptera: Meliponini). Apidologie, 
42(4): 499-507. doi: 10.1007/s13592-011-0013-0.

Francoy, T.M.; Faria Franco, F. \& Roubik, D.W. 2012. Integrated landmark and outline-based morphometric methods efficiently distinguish species of Euglossa (Hymenoptera, Apidae, Euglossini). Apidologie, 43(6): 609-617. doi: 10.1007/s13592-012-0132-2.

Freitas, F.V.; Santos Júnior, J.E.; Santos, F.R. \& Silveira F.A. 2018. Species delimitation and sex associations in the bee genus Thygater, with the aid of molecular data, and the description of a new species. Apidologie, 49(4):1-13. doi: 10.1007/s13592-018-0576-0.

Fujita, M.K.; Leaché, A.D.; Burbrink, F.R.; McGuire, J.A. \& Moritz, C. 2012. Coalescentbased species delimitation in an integrative taxonomy. Trends in Ecology and Evolution, 27(9): 480-488. doi: 10.106/j.tree.2012.04.012.

Galtier, N.; Nabholz, B.; Glémin, S. \& Hurst, G.D.D. 2009. Mitochondrial DNA as a marker of molecular diversity: a reappraisal. Molecular Ecology, 18(22): 4541-4550. doi: 10.1111/j.1365-294X.2009.04380.x.

Gibbs, J. 2009. Integrative taxonomy identifies new (and old) species in the Lasioglossum (Dialictus) tegulare (Robertson) species group (Hymenoptera, Halictidae). Zootaxa, 2032: 1-38.

Gibert, P.; Debat, V. \& Ghalambor, C.K. 2019. Phenotypic plasticity, global change, and the speed of adaptative evolution. Current Opinion in Insect Science, 35: 34-40. doi: 10.1016/j.cois.2019.06.007.

Glenn, T.C.; Pierson, T.W.; Bayona-Vásquez, N.J.; Kieran, T.J.; Hoffberg, S.L.; Thomas, I.V.J.C.; Lefever, D.E.; Finger, J.J.W.; Gao, B.; Bian, X.; Louha, S.; Kolli, R.T.; Bentley, K.; Rushmore, J.; Wong, K.; Shaw, T.I.; Rothrock, J.M.J.; McKee, A.M.; Guo, T.L.; Mauricio, R.; Molina, M.,; Cummings, B.S.; Lash, L.H.; Lu, K.; Gilbert, G.S.; Hubbell, S.P. \& Faircloth, B.C. 2019. Adapterama II: universal amplicon sequencing on Illumina platforms (TaggiMatrix). PeerJ, 7: (e7786):1-26. doi: 10.7717/peerj.7786.

Godoy, R.E.; Shimabukuro, P.H.F.; Santos, T.V., Pessoa, F.A. C.; Cunha, A.E.F.L.; Santos, F.K.M.; Vilela, M.L.; Rangel, E.F. \& Galati, E.A.B. 2018. Geometric morphometry of the head in sand flies (Diptera: Psychodidae: Phlebotominae), an alternative approach to taxonomy studies. Zootaxa, 4504: 566-576. doi: 10.11646/zootaxa.4504.4.7.

Goldstein, P.Z. \& DeSalle, R. 2010. Integrating DNA barcode data and taxonomic practice: determination, discovery, and description. Bioessays, 33(2): 135-147. doi: 
10.1002/bies.201000036.

Gonçalves, R.B. \& Melo, G.A.R. 2012. Phylogeny and revision of the bee genus Rhinocorynura Schrottky (Hymenoptera, Apidae, Augochlorini), with comments on its female cephalic polymorphism. Revista Brasileira de Entomologia, 56: 29-46. doi: 10.1590/S008556262012005000011.

González-Vaquero, R.A. \& Roig-Alsina A. 2019. The bee Ruizanthedella mutabilis Spinola (Hymenoptera: Halictidae): a very common but poorly known species studied using integrative taxonomy. Zootaxa, 4563 (1): 191-200. doi: 10.11646/zootaxa.4563.1.12.

Grab, H.; Branstetter, M.G.; Amon, N.; Urban-Mead, K.R.; Park, M.G.; Gibbs, J.; Blitzer, E.J.; Poveda, K.; Loeb, G. \& Danforth, B.N. 2019. Agriculturally dominated landscapes reduce bee phylogenetic diversity and pollination services. Science, Washington, 363(6424):282-284. doi: 10.1126/Science.aat6016.

Grando, C.; Amon, N.D.; Clough, S.J.; Guo, N.; Wei, W. Azevedo, P.; López-Uribe, M.M. \& Zucchi, M.I. 2018. Two colors, one species: the case of Melissodes nigroeanea (Apidae: Eucerini), an important pollinator of cotton fields in Brazil. Sociobiology, 65(4): 645653. doi: 10.13102/sociobiology.v65i4.3464.

Grassi-Sella, M.L.; Garófalo, C.A. \& Francoy, T.M. 2018. Morphological similarity of widely separated populations of two Euglossini (Hymenoptera: Apidae) species based on geometric morphometrics of wings. Apidologie, 49: 151-161. doi: 10.1007/s13592-0170536-0.

Gueuning, M.; Frey, J.E. \& Praz, C. 2020. Ultraconserved yet informative for species delimitation: UCEs resolve long-standing systematic enigma in Central European bees. Molecular Ecology, 29(21): 4203-4220. doi: 10.1111/mec.15629.

Guindon, S.; Dufayard, J.F.; Lefort, V.; Anisimova, M.; Hordijk, W. \& Gascuel, O. 2010. New algorithms and methods to estimate maximum likelihood phylogenies: assessing the performance of phyml 3.0. Systematic Biology, 59(3): 307-321. doi: 10.1093/sysbio/syq010.

Hebert, P.D.N.; Cywinska, A.; Ball, S.L. \& de Waard, J.R. 2003. Biological identifications through DNA barcodes. Proceedings of the Royal Society Biological Sciences, 270: 313-321. doi: 10.1098/rspb.2002.221.

Hebert, P.D.N; Stoeckle, M.Y.; Zemlak, T.S. \& Francis, C.M. 2004. Identification of birds 
through DNA barcodes. PLoS Biology, 2: e312. doi: 10.1371/journal.pbio.0020312.

Heled, J. \& Drummond, A.J. 2010. Bayesian inference of species trees from multilocus data. Molecular Biology and Evolution, 27(3): 570-580. doi: 10.1093/molbev/msp274.

Hickerson, MJ.; Meyer, C.P. \& Moritz, C. 2006. DNA barcoding will often fail to discover new animal species over broad parameter space. Systematic Biology, 55(5): 729-739. doi: $10.1080 / 10635150600969898$.

Hill, G.E. 2016. Mitonuclear coevolution as the genesis of speciation and the mitochondrial DNA barcode gap. Ecology and Evolution, 6(16): 5831-5842. doi: 10.1002/ece3.2338.

Hoang, D.T.; Chernomor, O.; von Haeseler, A.; Minh, B.Q. \& Vinh, L.S. 2018. UFBoot2: Imporving the ultrafast bootstrap approximation. Molecular Biology and Evolution, 35(2): 518-522. doi: 10.1093/molbev/msx281.

Huang, J.; Wu, J.; An, J. \& Williams, P.H. 2015. Newly discovered colour-pattern polymorphism of Bombus koreanus females (Hymenoptera: Apidae) demonstrated by DNA barcoding. Apidologie, 46(2): 250-261. doi: 10.1007/s13592-014-0319-9.

Hurtado-Burillo, M.; Jara, L.; May-Itzá, W.J.; Quezada-Euán, J.J.G.; Ruiz, C. \& Rúa, P. 2016. A geometric morphometric and microsatellite analyses of Scaptotrigona mexicana and $S$. pectoralis (Apidae: Meliponini) sheds light on the biodiversity of Mesoamerican stingless bees. Journal of Insect Conservation, 20(5): 753-763. doi: 10.1007/s10841-016-98991.

Jörger, K.M. \& Schrodl, M. 2013. How to describe a cryptic species? Practical challenges of molecular taxonomy. Frontiers in Zoology, 10(59):1-27 . doi: 10.1186/1742-9994-1059.

Kalyaanamoorthy, S.; Minh, B.Q.; Wong, T.K.F; von Haeseler, A. \& Jermiin, L.S. 2017. ModelFinder: Fast model selection for accurate phylogenetic estimates. Nature Methods, 14(6): 587-589. doi: 10.1038/nmeth.4285.

Katoh, K.; Rozewicki, J. \& Yamada, K.D. 2019. MAFFT online service: multiple sequence alignment, interactive sequence choice and visualization. Briefings in Bionformatics, 20(4): 1160-1166. doi: 10.1093/bib/bbx108.

Katoh, K. \& Standley, D.M. 2013. MAFFT multiple sequence alignment software version 7: improvements in performance and usability. Molecular Biology and Evolution, 30(4): 772-780. doi: 10.1093/molbev/mst010. 
Koch, J.B.; Rodriguez, J.; Pitts, J.P. \& Strange, J.P. 2018. Phylogeny and population genetic analyses reveals cryptic speciation in the Bombus fervidus species complex (Hymenoptera: Apidae). PLoS ONE, 13: e0207080. doi: 10.1371/journal.pone.0207080.

Kumar, S.; Stecher, G.; Li, M.; Knyaz, C. \& Tamura, K. 2018. Mega X. Molecular evolutionary genetics analysis across computing platforms. Molecular Biology and Evolution, 35: 1547-1549. doi: 10.1093/molbev/msy096.

Klingenberg, C.P. 2011. MorphoJ: an integrated software package for geometric morphometrics. Molecular Ecology Resources, 11(2): 353-357. doi: 10.1111/j.17550998.2010.02924.x.

Klingenberg, C.P. 2015. Analyzing fluctuating asymmetry with geometric morphometrics: concepts, methods, and applications. Symmetry-Basel, 7(2): 843-934. doi: $10.3390 /$ sym 7020843 .

Knowles, L.L. \& Carstens, B.C. 2007. Delimiting species without monophyletic gene trees. Systematic Biology, 56(6): 887-895. doi: 10.1080/10635150701701091.

Kress, W.J.; Wurdack, E.A.; Zimmer, L.A.; Weigt, L. \& Janzen, D.H. 2005. Use of DNA barcodes to identify flowering plants. Proceedings of the National Academy of Sciences, USA, 102(23): 8369-8374. doi: 10.1073/pnas.0503123102.

Lajus D.; Sukhikh N. \& Alekseev V. 2015. Cryptic or pseudocryptic: Can morphological methods inform copepod taxonomy? An analysis of publications and a case study of the Eurytemora affinis species complex. Ecology and Evolution, 5(12):2374-2385. doi: 10.1002/ece3.1521.

Lachenbruch, P.A. 1967. An almost unbiased method of obtaining confidence intervals for the probability of misclassification in discriminant analysis. Biometrics, 23(4): 639-645. doi: $10.2307 / 2528418$.

Lanfear, R.; Frandsen, P.B.; Wright, A.M.; Senfeld, T. \& Calcott, B. 2017. PartitionFinder 2: new methods for selecting partitioned models of evolution for molecular and morphological phylogenetic analyses. Molecular Biology and Evolution, 34(3): 772773. doi: 10.1093/molbev/msw2560.

Leaché, A.D. \& Fujita, M.K. 2010. Bayesian species delimitation in West African forest geckos (Hemidactylus fasciatus). Proceedings of the Royal Society Biological Sciences, 277(1697): 3071-3077. doi: 10.1098/rspb.2010.0662. 
Leliaert, F.; Verbruggen, H.; Vanormelingen, P.; Steen, F.; López-Bautista, J.M.; Zuccarello, G.C.; \& Clerck, O. 2014. DNA-based species limitation in algae. European Journal of Phycology, 49: 179-196. doi: 10.1080/09670262.2014.904524.

Lemmon, E.M.; Lemmon, A.R. 2013. High-throughput genomic data in systematics and phylogenetics. Annual Review of Ecology, Evolution and Systematics, 44:99-121. doi: 10.1146/annurev-ecolsys-110512-135822.

Lemmon, A.R.; Emme, S.A. \& Lemmon, E.M. 2012. Anchored hybrid enrichment for massively high-throughput phylogenomics. Systematic Biology, 61(5):727-744. doi: 10.1093/sysbio/sys049.

Lepeco, A. \& Gonçalves, R.B. 2018. The Colour and the shape: Morphological variation on a facultatively eusocial bee Augochlora (Augochlora) amphitrite (Schrottky). Sociobiology, 65(4 Special): 662-670. doi: 10.13102/sociobiology.v65i4.3388.

Longino, J.T. \& Branstetter, M.G. 2020. Phylogenomic species delimitation, taxonomy, and 'bird guide' identification for the Neotropical ant genus Rasopone (Hymenoptera: Formicidae). Insect Systematics and Diversity, 4(2): 1-33. doi: 10.1093/isd/ixaa004.

López-Uribe, M.M.; Zamudio, K.R.; Cardoso, C.F. \& Danforth, B.N. 2014. Climate, physiological tolerance and sex-biased dispersal shape genetic structure of Neotropical orchid bees. Molecular Ecology, 23(7): 1874-1890. doi: 10.1111/mec.12689.

Liu, L.; Yu, L.; Kubatko, L.; Pearl, D.K. \& Edwards, S.V. 2009. Coalescent methods for estimating phylogenetic trees. Molecular Phylogenetics and Evolution, 53(1): 320328. doi: 10.1016/j.ympev.2009.05.033.

Marques, M.F.; Hautequestt, A.P.; Oliveira, U.B.; Manhães-Tavares, V.F.; Perkles, O.R.; Zappes, C.A. \& Gaglianone, M.C. 2017. Local knowledge on native bees and their role as pollinators in agricultural communities. Journal of Insect Conservation, 21(2): 345356. doi: 10.1007/s10841-017-9981-3.

McCormack, J.E.; Harvey, M.G.; Faircloth, B.C.; Crawford, N.G.; Glenn, T.C. \& Brumfield, R.T. 2013. A phylogeny of birds based on over 1.500 loci collected by target enrichment and high-throughput sequencing. PLos ONE, 8: e54848. doi: 10.1371/journal.pone.0054848.

Melo, G.A.R. 2014. Notes on the systematics of the orchid bee genus Eulaema (Hymenoptera: Apidae). Revista Brasileira de Entomologia, 58(3): 235-240. doi: 10.1590/S0085- 
56262014000300003.

Michener, C.D. 2007. The Bees of the World. 2.ed. Baltimore, Johns Hopkins University Press.

Moczek, A.P. 2010. Phenotypic plasticity and diversity in insects. Philosophical Transactions of the Royal Society, 365(1540):593-603. doi: 10.1098/rstb.2009.0263.

Murray, T.E.; Fitzpatrick, Ú.; Brown, M.J.F. \& Paxton, R.J. 2008. Cryptic species diversity in a widespread bumble bee complex revealed mitochondrial DNA RFLPs. Conservation Genetics, 9(3): 653-666. doi: 10.1007/s 10592-007-9394-z.

Nguyen, L.T.; Schmidt, H.A.; von Haeseler, A. \& Minh, B.Q. 2015. IQ-TREE: A fast and effective stochastic algorithm for estimating maximum likelihood phylogenies. Molecular Biology and Evolution, 32(1): 268-274. doi: 10.1093/molbev/msu300.

Okonechnikov, K.; Golosova, O. \& Fursov, M. 2012. Unipro UGENE: a unified bioinformatics toolkit. Bioinformatics, 28(8): 1166-1167. doi: 10.1093/bioinformatics/bts091.

Oleksa, A. \& Tofilski, A. 2014. Wing geometric morphometrics and microsatellite analysis provide similar discrimination of honey bee subspecies. Apidologie, 46: 49-60. doi: 10.1007/s 13592-014-0300-7.

Oliveira, M.L. 2000. O gênero Eulaema Lepeletier, 1841 (Hymenoptera: Apidae: Euglossini): filogenia, biogeografia e relações com as Orchidaceae. PhD Thesis. Faculdade de Filosofia, Ciência e Letras, USP, Ribeirão Preto, SP. 159p.

Oliveira, M.L. 2006. Três novas espécies de abelhas da Amazônia pertencentes ao gênero Eulaema Lepeletier, 1841 (Hymenoptera: Apidae: Euglossini). Acta Amazonica, 36(1): 273-286. doi: 10.1590/S0044-59672006000100015.

Padial, J.M. \& De La Riva, I. 2007. Integrative taxonomists should use and produce DNA barcodes. Zootaxa, 1586: 67-68.

Padial, J.M.; Miralles, A.; De La Riva, I. \& Vences, M. 2010. The integrative future of taxonomy. Frontiers in Zoology, 7(16):1-14. doi: 10.1186/1742-9994-7-16.

Page, T. J.; Choy, S. C. \& Hughes, J. M. 2005. The taxonomic feedback loop: Symbiosis of morphology and molecules. Biology Letters, 1: 139- 142. doi: 10.1098/rsbl.2005.0298.

Pauly, A.; Noël, G.; Sonet, G.; Notton, D.G. \& Boevé, J-L. 2019. Integrative taxonomy resuscitates two species in the Lasioglossum villosulum complex (Kirby, 1802) (Hymenoptera: Apoidea: Halictidae). European Journal of Taxonomy, 541: 1-43. doi: 
10.5852/ejt.2019.541.

Pie, M.R.; Bornschein, M.R.; Ribeiro, L.F. Faircloth, B.C. \& McCormack. 2019. Phylogenomic species delimitation in microendemic frogs of the Brazilian Atlantic Forest. Molecular Phylogenetics and Evolution, 141(106627):1-8 .doi: 10.1016/j.ympev.2019.106627.

Pokorny, T.; Lunau, K.; Quezada-Euán, J.J.G. \& Eltz, T. 2014. Cuticular hydrocarbons distinguish cryptic sibling species in Euglossa orchid bees. Apidologie, 45(2): 276-283.

Quezada-Euán, J.J.G.; Paxton, R.J.; Palmer, K.A.; May-Itza, W.D.M.; Tay, W.T. \& Oldroyd, B.P. 2007. Morphological and molecular characters reveal differentiation in a Neotropical social bee, Melipona beecheii (Apidae: Meliponini). Apidologie, 38(3): 247-258.

Quezada-Euán, J. J. G.; Sheets, H. D.; de Luna, E. \& Eltz, T. 2015. Identification of cryptic species and morphotypes in male Euglossa: Morphometric analysis of forewings (Hymenoptera: Euglossini). Apidologie, 46(6): 787-795. doi: 10.1007/s 13592-015-03697.

Rambout, A. 2012. Figtree: tree figure drawing tool version 1.4.4. Available at: http://tree.bio.ed.ac.uk/software/figtree.

Ramos, K.S. \& Rozen, J.G. 2014. Psaenythisca, a new genus of bees from South America (Apoidea: Andrenidae: Protandrenini) with a description of the nesting biology and immature stages of one species. American Museum Novitates, 3800: 1-32. doi: $10.1206 / 3800.1$

Rannala, B.H. 2015. The art and science of species delimitation. Current Zoology, 61(5): 846853. doi: 10.1093/czoolo/61.5.846.

Rannala, B. \& Yang, Z. 2003. Bayes estimation of species divergence times and ancestral population sizes using DNA sequences from multiple loci. Genetics, 164(4):1645-1656.

Rocha-Filho, L.C.; Cerântola, N.C.M.; Garófalo, C.A.; Imperatriz-Fonseca, V.L. \& Del Lama, M.A. 2013. Genetic differentiation of the Euglossini (Hymenoptera, Apidae) populations on a mainland coastal plain and an island in southeastern Brazil. Genetica, 141(1-3): 6574. doi: 10.1007/s10709-013-9706-9.

Rocha-Filho, L.C. \& Garófalo, C.A. 2015. Males of the orchid bee Eulaema cingulata (Hymenoptera: Apidae) as important vectors of the cleptoparasitic beetle Meloetyphlus fuscatus (Coleoptera: Meloidae). Apidologie, 46(3): 286-291. doi: 10.1007/s13592-0140322-1. 
Rohland, N. \& Reich, D. 2012. Cost-effective, high-throughput DNA sequencing libraries for multiplexed target capture. Genome Resources, 22(5): 939-946.

Rohlf, F.J. 2006. TpsDig, 2.0. New York, Department of Ecology and Evolution, State University of at Stony Brook.

Rohlf, F.J. 2013. TpsUtil. New York, Department of Ecology and Evolution, State University of Stony Brook. http://life.bio.sunysb.edu/morph/.

Ronquist, F.; Teslenko, M.; Mark, P.V.D; Ayres, D.L.; Darling, A.; Höhna, S.; Larget, B. Liu, L.; Suchard, M.A. \& Huelsenbeck, J. 2012. MrBayes 3.2: Efficient Bayesian phylogenetic inference and model choice across a large model space. Systematic Biology, 61(3): 539-542. doi: 10.1093/sysbio/sys029.

Rubinoff, D.; Cameron, S. \& Will, K. 2006. A genomic perspective on the shortcomings of mitochondrial DNA for "barcoding” identification. Journal of Heredity, 97(6): 581-594.

Sáez, A.G. \& Lozano, E. 2005. Body doubles. Nature, 433: 111.

Sangster, G. 2018. Integrative taxonomy of birds: the nature and delimitation of species. In: Tietze, D.T. (Ed). Bird Species - How they arise, modify and vanish. New York, Spring. p. 9-37. doi: 10.1007/978-3-319-91689-7.

Santos, C.F.; Santos, P.D.S.; Marques, D.M., Costa, T. \& Blochtein. 2019. Geometric morphometrics of the forewing shape and size discriminate Plebeia species (Hymenoptera: Apidae) nesting in different substrates. Systematic Entomology, 44(4): 787-796. doi: 10.1111/syen.12354.

Sigovini, M.; Keppel, E. \& Tagliapietra, D. 2016. Open nomenclature in the biodiversity era. Methods in Ecology and Evolution, 7(10): 1217-1225. doi: 10.1111/2041-210X.12594.

Silva, F.L.; Grassi-Sella, M.L.; Francoy, T.M. \& Costa, A.H.R. 2015. Evaluating classification and feature selection techniques for honeybee subspecies identification using wing images. Computers and Electronics in Agriculture, 114: 68-77. doi: 10.1016/j.compag.2015.03.012.

Smith, B.T.; Harvey, M.G.; Faircloth, B.C.; Glenn, T.C. \& Brumfield, R.T. 2013. Target capture and massively parallel sequencing of ultraconserved elements (UCEs) for comparative studies at shallow evolutionary time scales. Systematic Biology, 63(1) 8395. doi: 10.1093/sysbio/syt061. 
Souza, D.A.; Wang, Y.; Kaftanoglu, O.; De Jong, D.; Amdam, G.V.; Gonçalves, L.S. \& Francoy, T.M. 2015. Morphometric identification of queens, workers and intermediates in In Vitro reared honey bees (Apis mellifera). PLoS ONE, 10: e0123663. doi: 10.1371/jounal.pone.0123663.

Sukumaran, J. \& Knowles, L.L. 2017. Multispecies coalescent delimits structure, not species. Proceedings of the National Academy of Sciences, 114(7): 1607-1612. doi: 10.1073/pnas.1607921114.

Tagliacollo, V. A. \& Lanfear, R. 2018. Estimating improved partitioning schemes for ultraconserved elements. Molecular Biology and Evolution, 35(7): 1798-1811. doi: 10.1093/molbev/msy069.

Takahata, N.; Satta, Y. \& Klein, J. 1995. Divergence time and population- size in the lineage leading to modern humans. Theoretical Population Biology, 48(2): 198-221. doi: 10.1006/tpbi.1995.1026.

Talavera, G. \& Castresana, J. 2007. Improvement of phylogenies after removing divergent and ambiguously aligned blocks from protein sequence alignments. Systematic Biology, 56(4): 564-577. doi: 10.1080/10635150701472164.

Via, S.; De Jong, G.; Scheiner, S.M.; Schlichting, C.D. \& Van Tienderen, P.H. 1995. Adaptative phenotypic plasticity: consensus and controversy. Trends in Ecology and Evolution, 10(5): 212-217. Adaptative phenotypic plasticity: consensus and controversy.

Young, A.D. \& Gillung, J.P. 2020. Phylogenomics - principles, opportunities and pitfalls of big-data phylogenomics. Systematic Entomology, 45(2): 225-247. doi: 10.111/syen.12406.

Ward, R.D.; Zemlak, T.S.; Innes, B.H.; Last, P.R. \& Hebert, P.D.N. 2005. DNA barcoding Australia's fish species. Philosophical Transactions of the Royal Society B, 360(1462): 1847-1857. doi: 10.1098/rstb.2005.1716.

Wheeler, Q.D. 2008. Undisciplined thinking: morphology and Hennig's unfinished revolution. Systematic Entomology, 33(1): 2-7. doi: 10.1111./j.1365-3113.2007.00411.x.

Williams, P.H.; Brown, M.J.F.; Carolan, J.C.; An, J.; Aytekin, A. M.; Best, L. R.; Byvaltsev, A.M.; Cederberg, B.; Dawson, R.; Huang, J.; Ito, M.; Monfared, A.; Raina, R.H.; SchmidHempel, P.; Scheffield, C.S.; Sima, P. \& Xie, Z. 2012. Unveiling cryptic species of the bumble bee subgenus Bombus s. str. worldwide with COI barcodes (Hymenoptera: 
Apidae). Systematics and Biodiversity, 10: 21-56. doi: 10.1080/14772000.664574.

Zhang, C.; Rabiee, M.; Sayyari, E. \& Mirarab, S. 2018. ASTRAL-III: polynomial time species tree reconstruction from partially resoled gene trees. BMC Bioinformatics, 19: 153. doi: 10.1186/s12859-018-2129-y.

Zhang, C.; Zhang, D-X.; Zhu, T. \& Yang, Z. 2011. Evaluation of a bayesian coalescent method of species delimitation. Systematic Biology, 60(6): 747-761. doi: 10.1093/sysbio/syr071. 